\title{
Multiple rings in the transition disk and companion candidates around $\mathrm{RX}$ J1615.3-3255
}

\section{High contrast imaging with VLT/SPHERE ${ }^{\star}, \star \star$}

\author{
J. de Boer ${ }^{1}$, G. Salter ${ }^{2}$, M. Benisty ${ }^{3}$, A. Vigan ${ }^{2}$, A. Boccaletti ${ }^{4}$, P. Pinilla ${ }^{1}$, C. Ginski ${ }^{1}$, A. Juhasz ${ }^{5}$, A.-L. Maire ${ }^{6}$,
}

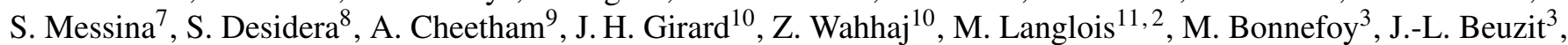
E. Buenzli ${ }^{6}$, G. Chauvin ${ }^{3}$, C. Dominik ${ }^{12}$, M. Feldt ${ }^{6}$, R. Gratton ${ }^{7}$, J. Hagelberg 9 , A. Isella ${ }^{13}$, M. Janson ${ }^{14}$, C. U. Keller ${ }^{1}$, A.-M. Lagrange ${ }^{3}$, J. Lannier ${ }^{3}$, F. Menard ${ }^{3}$, D. Mesa ${ }^{7}$, D. Mouillet ${ }^{3}$, M. Mugrauer ${ }^{15}$, S. Peretti ${ }^{9}$, C. Perrot ${ }^{4}$, E. Sissa ${ }^{7}$, F. Snik ${ }^{1}$, N. $\operatorname{Vogt}^{16}$, A. Zurlo ${ }^{17,2}$, and SPHERE Consortium

(Affiliations can be found after the references)

Received 6 July 2016 / Accepted 20 September 2016

\begin{abstract}
Context. The effects of a planet sculpting the disk from which it formed are most likely to be found in disks that are in transition between being classical protoplanetary and debris disks. Recent direct imaging of transition disks has revealed structures such as dust rings, gaps, and spiral arms, but an unambiguous link between these structures and sculpting planets is yet to be found.

Aims. We aim to find signs of ongoing planet-disk interaction and study the distribution of small grains at the surface of the transition disk around RXJ1615.3-3255 (RX J1615).

Methods. We observed RX J1615 with VLT/SPHERE. From these observations, we obtained polarimetric imaging with ZIMPOL ( $R^{\prime}$-band) and IRDIS $(J)$, and IRDIS $(H 2 H 3)$ dual-band imaging with simultaneous spatially resolved spectra with the IFS $(Y J)$.

Results. We image the disk for the first time in scattered light and detect two arcs, two rings, a gap and an inner disk with marginal evidence for an inner cavity. The shapes of the arcs suggest that they are probably segments of full rings. Ellipse fitting for the two rings and inner disk yield a disk inclination $i=47 \pm 2^{\circ}$ and find semi-major axes of $1.50 \pm 0.01^{\prime \prime}$ (278 au), $1.06 \pm 0.01^{\prime \prime}$ (196 au) and $0.30 \pm 0.01^{\prime \prime}$ (56 au), respectively. We determine the scattering surface height above the midplane, based on the projected ring center offsets. Nine point sources are detected between $2.1^{\prime \prime}$ and $8.0^{\prime \prime}$ separation and considered as companion candidates. With NACO data we recover four of the nine point sources, which we determine to be not co-moving, and therefore unbound to the system.

Conclusions. We present the first detection of the transition disk of RX J1615 in scattered light. The height of the rings indicate limited flaring of the disk surface, which enables partial self-shadowing in the disk. The outermost arc either traces the bottom of the disk or it is another ring with semi-major axis $\gtrsim 2.35^{\prime \prime}$ ( $435 \mathrm{au}$ ). We explore both scenarios, extrapolating the complete shape of the feature, which will allow us to distinguish between the two in future observations. The most attractive scenario, where the arc traces the bottom of the outer ring, requires the disk to be truncated at $r \approx 360 \mathrm{au}$. If the closest companion candidate is indeed orbiting the disk at 540 au, then it would be the most likely cause for such truncation. This companion candidate, as well as the remaining four, all require follow up observations to determine if they are bound to the system.
\end{abstract}

Key words. protoplanetary disks - planet-disk interactions - circumstellar matter - stars: pre-main sequence - panets and satellites: detection planets and satellites: formation

\section{Introduction}

The evolution of circumstellar disks around pre-main sequence stars, and planet-disk interactions constitute two of the major components in our understanding of planet formation. Determining the disk structure (such as its geometry, gas and dust distribution) is essential to our understanding of both disk evolution and planet-disk interactions. Direct imaging of protoplanetary disks shows a variety of features, such as inner cavities, gaps, rings and spirals that can be observed both in early

* Based on observations made with ESO Telescopes at the La Silla Paranal Observatory under programme IDs 095.C-0298(A), 095.C0298(B), and 095.C-0693(A) during guaranteed and open time observations of the SPHERE consortium, and on NACO observations: program IDs: 085.C-0012(A), 087.C-0111(A), and 089.C-0133(A).

$\star \star$ The reduced images as FITS files are only available at the CDS via anonymous ftp to cdsarc.u-strasbg. fr (130.79.128.5) or via http://cdsarc.u-strasbg.fr/viz-bin/qcat?J/A+A/595/A114
(HL Tau; $\leq 1$ Myr, ALMA Partnership et al. 2015) and late evolutionary phases (e.g. HD 100546, 10 Myr, Grady et al. 2001). This leads us to question whether the shapes and features of protoplanetary disks are caused by the presence of planets, or if the disk structures are regulating the formation of planets, or both. If the disk structures are not created by nearby planets, we still need to determine what did create these structures.

Strom et al. (1989) classified a subset of protoplanetary disks by a dip in the infra-red (IR) range of their spectral energy distribution (SED), caused by an inner dust depleted region (cavity). The authors suggested these disks represent an evolutionary stage "in transition" between classical (younger) protoplanetary disks and more evolved debris disks. Multiple explanations have been suggested for the origin of cavities within transition disks, such as photo-evaporation (e.g. Hollenbach \& Gorti 2005), and clearing by massive planets (e.g. Strom et al. 1989; Alexander \& Armitage 2009; Pinilla et al. 2015). Transition disks also display other potential tracers 
Table 1. SPHERE observations of RX J1615.

\begin{tabular}{|c|c|c|c|c|c|c|c|c|c|c|c|}
\hline Date & Instrument & Mode & Coronagraph & Fixlter & $\lambda_{0}(\mu \mathrm{m})$ & $F W H M^{1}(\mathrm{~nm})$ & DIT (s) & $t_{\text {tot }}(\min )$ & Seeing (") & $S R^{2}(\%)$ & $F W H M^{3}$ (mas) \\
\hline $12-05-2015$ & IRDIS & IRDIFS & ALC_YJH_S & $H 2 H 3$ & $1.59 \& 1.67$ & $53 \& 55$ & 64 & 136.5 & 0.65 & $65-70$ & $44.5 \& 46.6$ \\
\hline $12-05-2015$ & IFS & IRDIFS & ALC_YJH_S & $Y J$ & $0.96-1.34$ & 55.1 & 64 & 136.5 & 0.65 & $45-65$ & $30.0-36.1$ \\
\hline $15-05-2015$ & IRDIS & IRDIFS & ALC_YJH_S & $H 2 H 3$ & $1.59 \& 1.67$ & $53 \& 55$ & 64 & 68.3 & 0.65 & $65-70$ & $44.5 \& 46.6$ \\
\hline $15-05-2015$ & IFS & IRDIFS & ALC_YJH_S & $Y J$ & $0.96-1.34$ & 55.1 & 64 & 68.3 & 0.65 & $45-65$ & $30.0-36.1$ \\
\hline 06-06-2015 & IRDIS & DPI & ALC_YJH_S & $J$ & 1.26 & 197 & 64 & 76.8 & 1.3 & $30-35$ & 42.5 \\
\hline 09-06-2015 & ZIMPOL & $\mathrm{P} 2$ & - & $R^{\prime}$ & 0.626 & 149 & 120 & 96 & 1.0 & $<3$ & 95.5 \\
\hline
\end{tabular}

Notes. ${ }^{(1)}$ Full width at half maximum (FWHM) of the filter transmission. For the IFS, the FWHM is given per spectral channel. ${ }^{(2)}$ Strehl ratios (SRs) are measured using the unsaturated point spread functions (PSFs) at the observing wavelength (Girard et al., in prep.). For the low level of AO correction obtained for ZIMPOL, the FWHM is the appropriate image quality metric. ${ }^{(3)}$ FWHM of the PSF.

of planet-disk interaction: the large scale structures in disks such as spiral arms in for example MWC 758 (Grady et al. 2013; Benisty et al. 2015); ring structures, as seen in RX J16042130 (Mayama et al. 2012; Pinilla et al. 2015) and TW Hydrae (Andrews et al. 2016; van Boekel et al. 2016) and dust trapping vortices (e.g., IRS 48, van der Marel et al. 2013).

The pre-main sequence star RXJ1615.3-3255, or 2MASS J16152023-3255051 (hereafter RXJ1615) is a member of the Lupus star forming region at a distance $d=185 \mathrm{pc}$ (Krautter et al. 1997). From its very strong $\mathrm{H}_{\alpha}$ emission, Wahhaj et al. (2010) determine that this $1.4 \mathrm{Myr}$ old star of $1.1 M_{\odot}$ is a Classical T Tauri Star (CTTS). The authors classify this target as having an IR-excess with a "Turn-on wavelength $(\lambda)$ in the range $(3.6-8 \mu \mathrm{m})$ of the IR Array Camera" (TIRAC, where turnon $\lambda$ is the smallest $\lambda$ with disk emission distinguishable from stellar emission). Wahhaj et al. (2010) describe TIRAC targets as young $(1.3 \pm 0.3 \mathrm{Myr})$ objects with a small inner clearing in the disks (i.e., transition disks). Andrews et al. (2011) have resolved the disk with the SMA interferometer at $880 \mu \mathrm{m}$, determined a disk position angle $\mathrm{PA}=143^{\circ}$, inclination $i=41^{\circ}$ and found in their best-fit model a low-density cavity in the dust disk within $r_{\text {cav }}=30 \mathrm{au}$. The disk is resolved with ALMA (cycle 0) in ${ }^{12} \mathrm{CO}(6-5)$ and $690 \mathrm{GHz}$ continuum data by van der Marel et al. (2015). The authors find $\mathrm{PA}=153^{\circ}, i=45 \pm 5^{\circ}$ and a dust cavity inside $r_{\text {cav }}=20$ au from their model. Both Andrews et al. (2011) and van der Marel et al. (2015) have used a gas-to-dust mass ratio of 100:1 in their models for the outer disk, and find a total disk mass of $0.128 M_{\odot}$ and $0.474 M_{\odot}$, respectively.

While sub-mm observations probe emission of the mm-sized grains in the deeper layers of the disk, the surface of the micron sized dust disk can be detected by its scattering of near-IR (NIR) starlight. The large contrast between starlight and disk surface brightness at NIR wavelengths requires us to remove the stellar speckle halo, in order to detect the light scattered by the disk.

The Spectro-Polarimetric High-contrast Exoplanet REsearch (SPHERE, Beuzit et al. 2008) instrument has been in commission at the Very Large Telescope (VLT) since 2014. SPHERE is an extreme adaptive optics (AO) assisted instrument designed for high-contrast imaging of young giant exoplanets and circumstellar disks.

We use SPHERE to image the disk of RX J1615 in scattered light at multiple wavelengths with the aim of constraining the 3D disk geometry. In addition, we perform a search for possible companions that could be responsible for sculpting the disk. We report the new observations and the data reduction in Sects. 2 and 3, respectively. Section 4 describes the new detections of the disk structures and companion candidates. In Sect. 5, we give constraints on the disk geometry and discuss possible scenarios for the disk vertical structure. We list our conclusions in
Sect. 6. Based on archival data, we study the stellar properties of RX J1615 in Appendix A.

\section{Observations}

\subsection{VLT/SPHERE IRDIS, IFS and ZIMPOL}

We observed RX J1615 at multiple wavelengths using different modes of SPHERE (listed in Table 1). The extreme AO system, SAXO (SPHERE AO for eXoplanet Observation, Fusco et al. 2014) includes a $41 \times 41$-actuator deformable mirror, pupil stabilization, differential tip tilt control and stress polished toric mirrors (Hugot et al. 2012) for beam transportation to the coronagraphs (Boccaletti et al. 2008; Martinez et al. 2009) and science instruments. The latter comprise a near-IR Dual-band Imager and Spectrograph (IRDIS, Dohlen et al. 2008), a near-infrared Integral Field Spectrometer (IFS, Claudi et al. 2008), and the Zurich IMaging POLarimeter (ZIMPOL, Thalmann et al. 2008).

The SpHere INfrared survey for Exoplanets (SHINE), executed during the guaranteed time observations (GTO) of the SPHERE consortium, includes observations of RXJ1615 on May 12 and 15 of 2015. The observations were performed with the IRDIFS mode (Zurlo et al. 2014) in pupil tracking, using IRDIS in dual-band imaging mode (Vigan et al. 2010) in the $H 2 H 3$ bands and IFS in $Y J$-band. The observations are taken with the Apodized pupil Lyot Coronagraph ALC_YJH_S, which has a diameter of 185 mas. During the May 12 observations the field rotated by $113.2^{\circ}$. On May 15 , we observed the system with a field rotation of $75.3^{\circ}$.

On June 6, 2015, the SPHERE-Disk GTO program observed RX J1615 with IRDIS in dual-band polarimetric imaging mode (DPI, Langlois et al. 2014; de Boer et al., in prep.) in $J$-band with the ALC_YJH_S coronagraph. For the six polarimetric cycles, we observed with the half-wave plate (HWP) at the angles $\theta_{\text {hwp }}=0^{\circ}, 45^{\circ}, 22.5^{\circ}$, and $67.5^{\circ}$ to modulate the linear Stokes parameters $(Q$ and $U)$. Per $\theta_{\text {hwp }}$ we have taken three exposures.

During open time observations on June 9, 2015 we observed RX J1615 with SPHERE's visible light polarimeter, ZIMPOL in field tracking (P2) non-coronagraphic mode in $R^{\prime}$-band with the dichroic beamsplitter. We recorded six polarimetric (or HWP) cycles, with $\theta_{\mathrm{hwp}}=0^{\circ}, 45^{\circ}, 22.5^{\circ}$, and $67.5^{\circ}$ to modulate $Q$ and $U$. For each $\theta_{\text {hwp }}$ two frames were recorded for each of the two detectors of ZIMPOL.

The faint guide star $(R=11.21 \mathrm{mag}$, Makarov 2007) poses a challenge for the AO system. Especially in the visible light which is split between ZIMPOL and the SAXO wave front sensor, the moderate seeing $\left(\sim 1.0^{\prime \prime}\right)$ yielded very poor Strehl ratio $S R_{R^{\prime}}<3 \%$ and $F W H M_{R^{\prime}}=95.5 \pm 1.5$ mas. 


\subsection{VLT/NACO and Keck/NIRC2 SAM}

Additionally, RXJ1615 had already been observed with VLT/NACO (Lenzen et al. 2003; Rousset et al. 2003) as part of a survey for substellar companions in the Lupus star forming region for three epochs on May 7th 2010, May 8th 2011 and August 7th 2012 (Mugrauer et al., in prep.). The system was imaged in the $K_{\mathrm{s}}$-band $\left(\lambda_{0}=2.18 \mu \mathrm{m}, \Delta \lambda=350 \mathrm{~nm}\right)$ in standard jitter imaging (field stabilized) mode. NACO's cube mode was used to save each individual frame (Girard et al. 2010). The detector integration time (DIT) for a single exposure was $1 \mathrm{~s}$ for the three epochs.

To investigate the possibility of stellar or high-mass substellar companions at close separations, we include the results from archival Keck NIRC2 sparse aperture masking (SAM) data of RX J1615. The target was observed on April 4, 2012 with DIT $=5 \mathrm{~s}$, at 4 coadds per frame, and for 12 frames in total; on July 8, 2012 with DIT $=5 \mathrm{~s}, 4$ coadds per frame, 62 frames in total; and on June 10, 2014 with DIT $=10 \mathrm{~s}, 2$ coadds per frame, 40 frames in total. In all three epochs, the $K^{\prime}$ filter and the nine hole mask were used.

\section{Data reduction}

\subsection{Reduction of the ZIMPOL P2 $R^{\prime}$-band data}

The polarimetric differential imaging (PDI, Kuhn et al. 2001) reduction of the ZIMPOL data was performed according to the description of de Boer et al. (2016) which is briefly summarized below. After dark subtraction and flat-fielding, we separated the orthogonal polarization states from each frame, and binned the resulting images to a pixel scale of $15 \times 15$ mas. For the first $\theta_{\text {hwp }}=0^{\circ}$, we obtained the intensity image $I_{Q^{+}}$by adding the two polarization states per frame, and summed over the two frames (which measure the " 0 " and " $\pi$ " phase, Schmid et al. 2012). We computed $Q^{+}$by subtracting the two polarization states per frame and subtracting the residual for the $\pi$-phase (2nd frame) from the residual image of the 0-phase (1st frame). Similarly, we measured $I_{Q^{-}}$and $Q^{-}$with $\theta_{\text {hwp }}=45^{\circ} ; I_{U^{+}}$and $U^{+}$with $\theta_{\mathrm{hwp}}=22.5^{\circ}$; and $I_{U^{-}}$and $U^{-}$with $\theta_{\mathrm{hwp}}=67.5^{\circ}$.

We centered each Stokes $I$ image by determining the offset between the image and a Moffat function using a crosscorrelation. We used this offset to shift the image to the center and apply the same shift to the corresponding $Q^{+/-}$and $U^{+/-}$images, and compute for both detectors $(\mathrm{d} 1 / \mathrm{d} 2)$ :

$Q_{\mathrm{d} 1 / \mathrm{d} 2}=\left(Q^{+}-Q^{-}\right) / 2$,

$U_{\mathrm{d} 1 / \mathrm{d} 2}=\left(U^{+}-U^{-}\right) / 2$.

Because we used the same $\left(R^{\prime}\right)$ filter for both ZIMPOL detectors, we obtained the final $Q, U$ and $I_{Q / U}$ images according to:

$Q=\left(Q_{\mathrm{d} 1}-Q_{\mathrm{d} 2}\right) / 2$,

$U=\left(U_{\mathrm{d} 1}-U_{\mathrm{d} 2}\right) / 2$,

where the minus sign was necessary because the polarizing beamsplitter yields orthogonal polarization signals for the two detectors. We corrected the $Q$ and $U$ images for remaining instrumental and background polarization according to Canovas et al. (2011).

Finally, we computed the azimuthal Stokes components (cf. Schmid et al. 2006, where the azimuth $\phi$ is defined with respect to the star-center):

$Q_{\phi}=Q \times \cos 2 \phi+U \times \sin 2 \phi$,

$U_{\phi}=Q \times \sin 2 \phi-U \times \cos 2 \phi$.
For cases of single scattering or simple symmetries, $Q_{\phi}$ should contain all the signal, while $U_{\phi}$ provides an indication of the measurement error. The $Q_{\phi}$ and $U_{\phi}$ images are smoothed with a boxcar of three pixel width and shown in Figs. 1a and d, respectively.

\subsection{Reduction of the IRDIS/DPI J-band data}

The IRDIS DPI mode splits the beam before two orthogonal polarizers create the two orthogonal images simultaneously on different halves of the detector: the ordinary intensity image $I_{\mathrm{o}}=$ $0.5(I+Q)$ and the extraordinary intensity image $I_{\mathrm{e}}=0.5(I-Q)$. After we subtracted the dark, and flat-fielded the images, we centered the image of both detector halves on a Moffat function, using a cross-correlation. This first centering does not guarantee that the star is placed exactly at the center yet, but does ensure that all images of the star are at the same place with respect to the center, which suffices to subtract the two orthogonal polarization states on each frame. For the first $\theta_{\text {hwp }} Q^{+}$was computed by subtracting the two simultaneouly measured beams:

$Q^{+}=\left.\left(I_{\mathrm{o}}-I_{\mathrm{e}}\right)\right|_{\theta_{\mathrm{hwp}}=0}$.

By performing this operation for each of the three frames observed per $\theta_{\text {hwp }}$ we obtained three $Q^{+} ; Q^{-} ; U^{+}$; and $U^{-}$images for $\theta_{\text {hwp }}=0^{\circ} ; 45^{\circ} ; 22.5^{\circ}$; and $67.5^{\circ}$, respectively.

We stacked the three difference-images obtained per $\theta_{\text {hwp }}$ and for each of the six HWP cycles applied the double difference:

$Q=\left(Q^{+}-Q^{-}\right) / 2$,

$U=\left(U^{+}-U^{-}\right) / 2$.

For all HWP cycles we applied a correction on the $Q$ and $U$ images to remove a detector artefact that creates continuous vertical bands on the IRDIS detector which vary with time. This correction is similar to the correction of Avenhaus et al. (2014) for comparable artefacts on the NACO detector: for each pixel column we took the median over the top 20 and bottom 20 pixels and subtracted this signal from the entire column. Next, we computed $Q_{\phi}$ and $U_{\phi}$ according to Eqs. (5) and (6).

An additional centering per HWP cycle uses a minimization of the signal in the $U_{\phi}$ image, which is based on the assumption that no astrophysical signal is measured in the $U_{\phi}$ image, only noise (including reduction artefacts). We shifted the $Q$ and $U$ image at subpixel steps in the $x$ and $y$ direction and compute $U_{\phi}$ for each step. The shift that has the $U_{\phi}$ image with the lowest value over a centered but co-shifted annulus ( $10 \leq r \leq 20$ pixels) is assumed to place the star closest to the center of the image.

After we stacked the centered $Q$ and $U$ images, we corrected for instrumental and sky polarization, as we did for the ZIMPOL reduction. From these stacked images, we computed the final $Q_{\phi}$ and $U_{\phi}$ images for the $J$-band, which are shown at the same intensity scale in Figs. 1b and e, respectively. Figures 1c and $\mathrm{d}$ show these $Q_{\phi}$ and $U_{\phi}$ images after scaling with the inclination-corrected radius squared, for which we used $i=45$ from van der Marel et al. (2015).

\subsection{Reduction of the IRDIS (H2H3) and IFS (YJ) pupil tracking data}

The IRDIS and IFS data are reduced using the SPHERE Data Center. We used the SPHERE pipeline (Pavlov et al. 2008) to process cosmetic reductions including sky subtraction, bad pixel removal, flat field and distortion corrections, IFS wavelength 


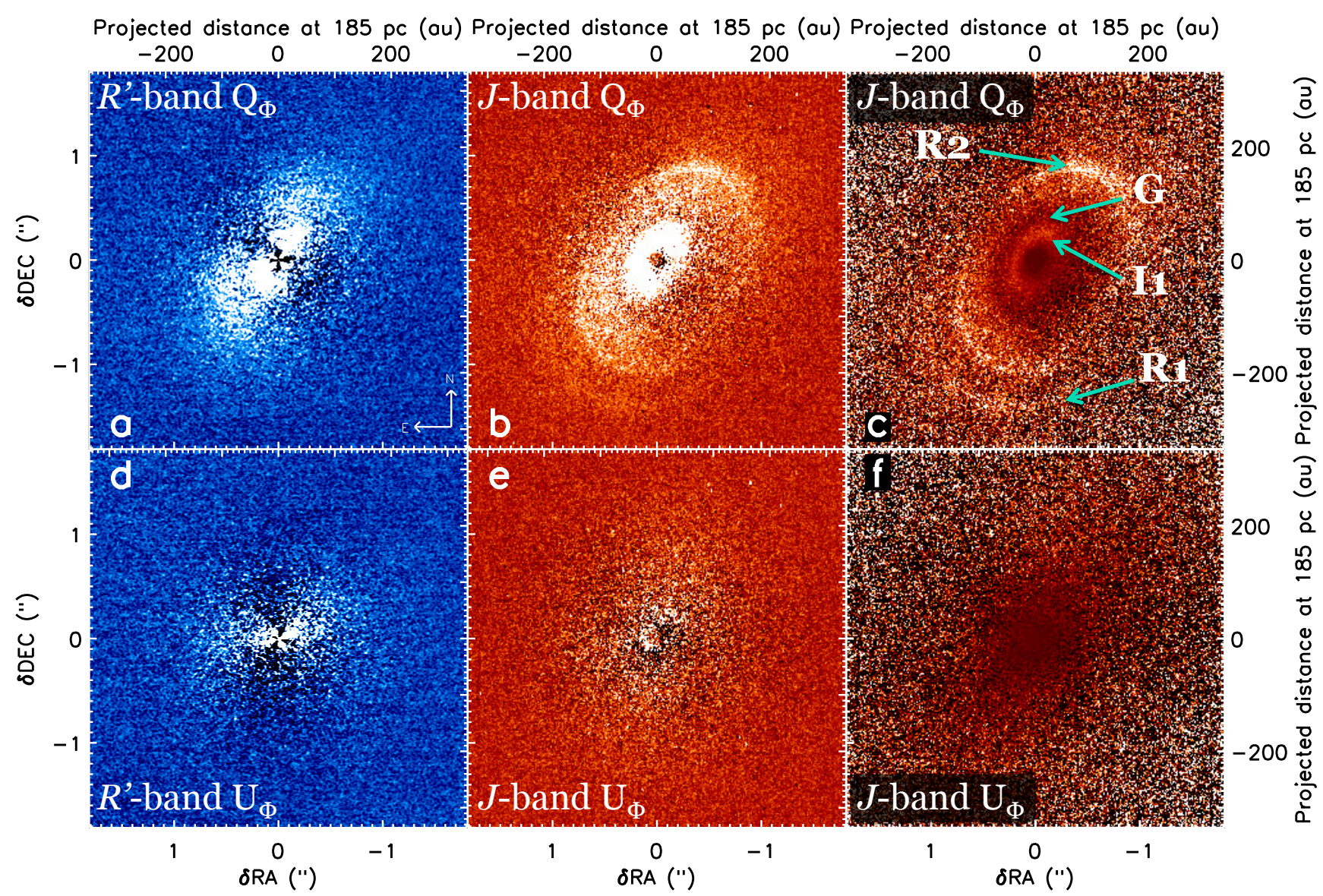

Fig. 1. a) $Q_{\phi}$ image of the ZIMPOL $R^{\prime}$-band data, smoothed with a boxcar of three pixels. No radial scaling is applied. b) $Q_{\phi}$ image of the IRDIS/DPI $J$-band data, without radial scaling. c) Same reduction as shown in panel b), with an inclination $\left(i=45^{\circ}\right)$ corrected $r^{2}$ scaling. This panel has the following disk features annotated: The southern ansa of a ring $(R 1)$, a full ring $R 2$, a clear dip in the surface brightness $(G)$ and an inner disk structure $I 1$. Panels d)-f) display the $U_{\phi}$ images corresponding to and with the same dynamic range as the $Q_{\phi}$ images of panels a)-c) respectively.

calibration, IFU flat correction, instrument anamorphism correction $(0.60 \pm 0.02 \%$, Maire et al. 2016), and frame registering.

Then, several types of angular differential imaging (ADI, Marois et al. 2006) based algorithms were implemented in a dedicated tool (SpeCal, Galicher et al., in prep.) to perform starlight subtraction independently for each of the two IRDIS filters, and the 39 IFS spectral chanels.

For IRDIS, we present the results obtained with classical ADI (cADI) for the full field of view (Fig. 4b) and the Template Locally Optimized Combination of Images algorithm (TLOCI, Marois et al. 2014) for a $4 " \times$ 4" field (Fig. 2). The May 12 data shows a strong vertical negative residual in the reduction, for both IRDIS (Fig. 2a) and IFS. We ascribe this to an aberration of the PSF, due to a loss of AO performance when coping with high altitude winds. The May 15 data does not suffer this effect. Figure 3 shows the IFS TLOCI+ADI reductions for May 15, where the first three panels show the median combination of 13 spectral chanels between $0.96-1.07 \mu \mathrm{m}, 1.08-1.21 \mu \mathrm{m}$, and $1.22-1.33 \mu \mathrm{m}$, respectively. The right-hand panel shows the median combination of all 39 spectral bands.

\subsection{Reduction of the NACO $K_{s}$ jitter imaging data}

The NACO data reduction is similar for all epochs. First, we averaged all frames in each data cube, and used the jitter routine in the ESO Eclipse package (Devillard 2001) to flat-field, shift and combine all averaged images. An initial offset of each frame was determined from the image header and was then refined using jitter's cross correlation routine. Since the star was moved to different positions on the detector, each pair of consecutive frames can be used to estimate the sky background in $K_{\mathrm{s}}$-band and subtract it. To remove the stellar halo, we stacked the centered image, and subtracted the same image after we had rotated it $180^{\circ}$. We present the resulting $180^{\circ}$ differential image in Fig. 4a.

The astrometric calibration of the NACO epochs was taken from Ginski et al. (2014). They imaged the core of the globular cluster 47 Tuc for this purpose in the same filter and imaging mode. These calibrations are within two days of the RX J1615 observations for the 2010 and the 2012 epoch. Due to bad conditions during the 2011 observations, no companion candidates were recovered.

\subsection{Reduction of the Keck NIRC2 SAM K' data}

Data were reduced using the aperture masking pipeline developed at the University of Sydney. An in-depth description of the reduction process can be found in Tuthill et al. (2000) and Kraus et al. (2008), but a brief summary follows: data were dark subtracted, flat-fielded, cleaned of bad pixels and cosmic rays, then windowed with a super-Gaussian function. The complex visibilities were extracted from the cleaned cubes and turned into 
J. de Boer et al.: SPHERE imaging of the disk and companion candidates around RXJ1615

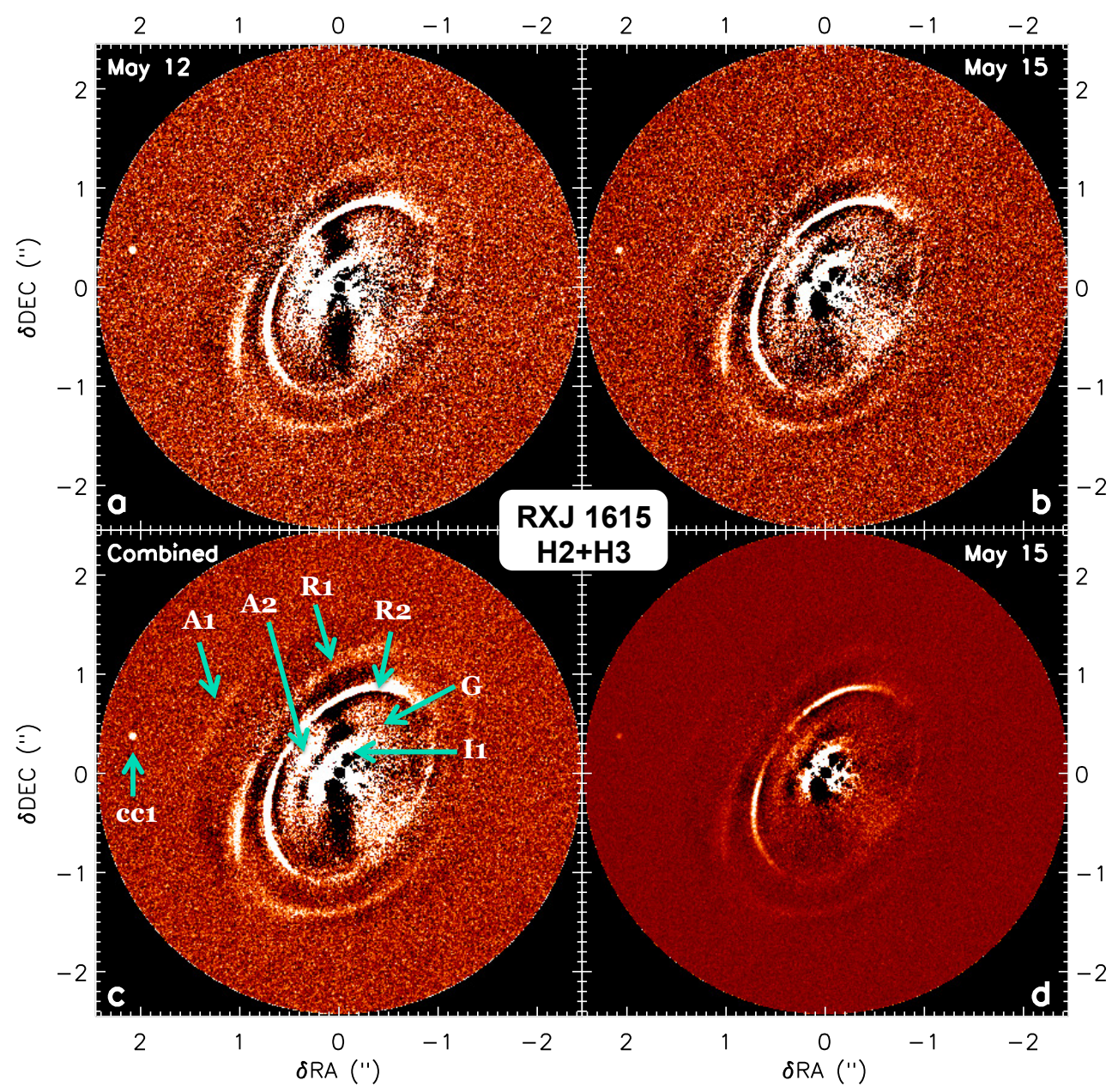

Fig. 2. TLOCI reductions of SPHERE-IRDIS ADI H2H3 (mean combination of the H2 and H3 filters) data. a) May 122015 ; b) May 15 2015; c) mean combination of May 12 and May 15, with features annotated. From the outside in, we see a point source, which we consider as a companion candidate (cc1), an arc (A1), two full rings $(R 1 \& R 2)$, another arc $(A 2)$, the location of the gap seen in Fig. 1c $(G)$, and an innermost disk structure (I1); d) same as b, for a $10 \times$ larger dynamic range.

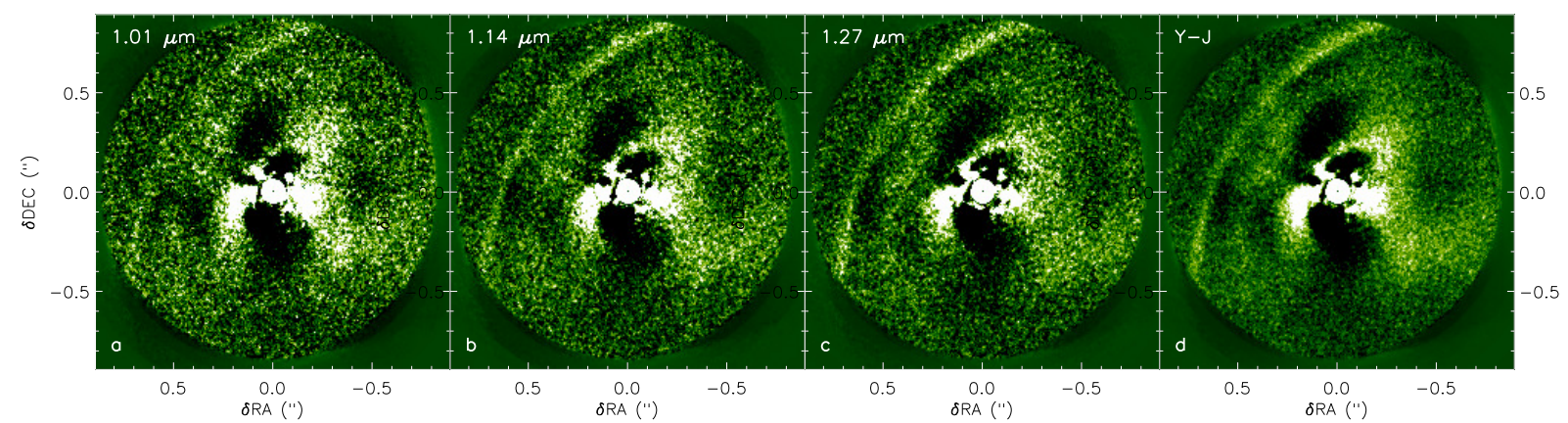

Fig. 3. TLOCI reductions of SPHERE-IFS ADI $Y-J$ data $(0.96-1.07 \mu \mathrm{m})$. a) Median of 13 channels $(0.96-1.07 \mu \mathrm{m})$. b) Median of 13 channels $(1.08-1.21 \mu \mathrm{m})$. c) Median of 13 channels $(1.22-1.33 \mu \mathrm{m})$. c) The median over the entire $Y-J$ range of the IFS.

closure phases. The closure phases were then calibrated by subtracting a weighted average of those measured on several pointsource calibrator stars observed during the same night.

\section{Results}

\subsection{Disk}

The disk of RX J1615 is detected in all the datasets included in this study. For the ADI as well as the PDI images, all features described below are brighter on the northeastern side from the major axis.

- Outer rings: in the $J$-band $r^{2}$ scaled $Q_{\phi}$ image (Fig. 1c) and the (not $r^{2}$ scaled) $H 2 H 3$ TLOCI ADI images of the disk, we see multiple arc-like and continuous ellipses that we consider to be ring-like features in the scattering $(\tau=1)$ surface of the disk, projected with the inclination of the system. For decreasing separation, Fig. 2c shows: an arc (A1); two full rings $R 1$ and $R 2$; a second arc (A2). Where the two arcs cross the minor axis of the disk, they seem to lie parallel 


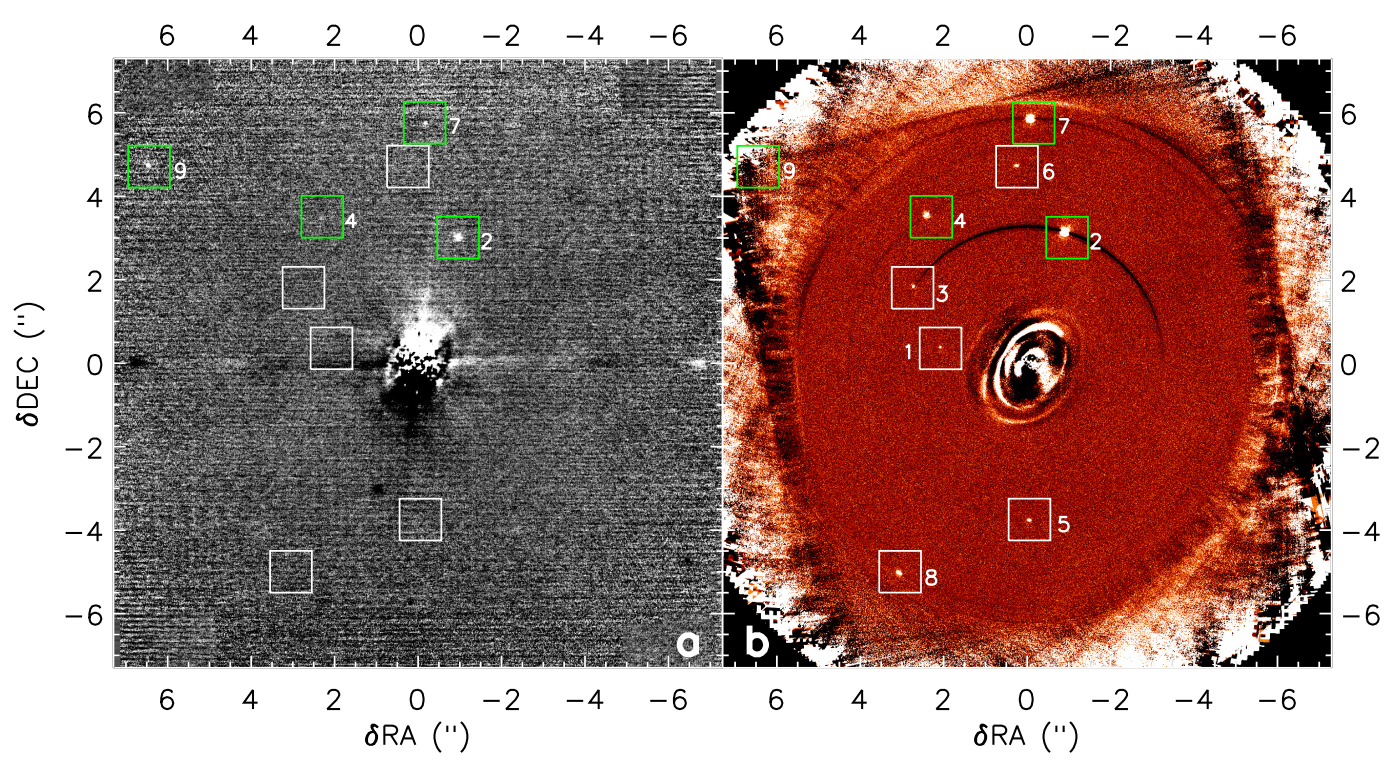

Fig. 4. Companion candidates detected in NACO and SPHERE data. a) NACO image in $K_{\mathrm{s}}$-band of May 7, 2010. We self-subtracted the image after it was rotated by $180^{\circ}$. A small section of $R 2$ is seen west of the star. b) SPHERE-IRDIS cADI in $H 23$ band of May 15,2015 . The green squares in a) and b) show the four point sources first detected with NACO; the white squares highlight the five point sources detected with SPHERE/IRDIS only.

to both rings. We therefore consider it most likely that both arcs are segments of full rings. The $A 2$ feature does not appear to be as clearly separated from $R 2$ in the $J$-band $r^{2}$ scaled polarization image, and is therefore not annotated in the image. However, in this $Q_{\phi}$ image the disk signal at radii $r \sim r(A 2)$ appears continuous in the azimuthal direction, which confirms that $A 2$ is indeed a full ring. The $R 2$ disk feature is detected (at least as a ring segment) in all datasets except the ZIMPOL $R^{\prime}$-band polarization image. In Fig. 3, we see that the $R 2$ segment which lies within the IFS field of view is detected more clearly at longer wavelengths. Only the southern ansa of $R 1$ is detected in the $J$-band $Q_{\phi}$ image. In Figs. 1b, c and 2 we can discern that $R 1$ and $R 2$ are clearly not concentric with the inner disk component $I 1$ and the star-center.

- Gap: a dip in the surface brightness or gap (feature $G$ ) in between features $A 2$ and $I 1$ is detected, most clearly in Fig. 1c. Figure 2c shows the gap as well, but not for all azimuth angles.

- Inner disk: closest to the star, we see an elliptical inner disk component (feature $I 1$ in Fig. 2b). The surface brightness is continuous inward in the $Q_{\phi}$ images in both $R^{\prime}$ and $J$-bands (Figs. 1a and b). However, for a disk with continuous surface density and a linearly increasing scattering surface we would expect the surface brightness to drop off with the distance to the star squared. Conversely, if we create an image with the surface brightness scaled with $r^{2}$ (corrected for the inclination), the previous example of a continuous surface density would show a continuous surface brightness. However, $I 1$ in our inclination-corrected $r^{2}$ scaled $J$-band image (Fig. 1c) appears more ring-like with a cavity inside, which agrees with the outer radius of the inner dust cavity as determined from the $880 \mu \mathrm{m}$ interferometric observations of Andrews et al. $\left(2011, r_{\text {cav }}=30 \mathrm{au}\right)$. Still, we consider the detection of the cavity as tentative because $r_{\text {cav }}$ is bordering the coronagraph. We also see a non-continuous surface brightness of $I 1$ in the ADI reductions of Fig. 2. However, observations of a disk with continuous surface brightness is likely to be afflicted by self-subtraction, which is often seen in ADI for low and intermediate inclination circumstellar disks (Milli et al. 2012). This kind of self-subtraction does not occur in PDI, which is extremely efficient at isolating the polarized disk signal, hence revealing the disk structure with high fidelity. We note that based on our results, we cannot rule out a disk component at a separation $r<30$ au (inside I1). However, Andrews et al. (2011) mention that their model requires a very low density inside $r_{\text {cav }}=30$ au to adequately fit the SED.

\section{Ellipse fitting}

For both the $J$-band PDI image and the H23-band TLOCI ADI image we have fit ellipses ${ }^{1}$ to the two rings and to the inner disk. The resulting ellipses are overplotted in Figs. 5a and b, and the ellipse parameters are listed in Table 2. From the assumption that the ellipses trace the highest surface above the midplane for circular rings (i.e., not the slope facing the star/wall), we determine the inclination of each ellipse according to $\cos i=$ semiminor axis/semi-major axis. With a weighted mean we find an inclination of the disk $i=47.3 \pm 0.4^{\circ}$, where the error represents the random error on the fit ellipses but does not include the systematic errors from our method. A conservative estimate of the systematic errors brings us to a final value of $i=47 \pm 2^{\circ}$, which is in good agreement with the inclination derived by van der Marel et al. (2015). The weighted mean for the position angles is $\mathrm{PA}=145.8 \pm 0.5^{\circ}$. Including our estimate of the systematic errors gives a final $\mathrm{PA}=146 \pm 2^{\circ}$, which is in-between the values of Andrews et al. $\left(2011, \mathrm{PA}=143^{\circ}\right)$ and van der Marel et al. $\left(2015, \mathrm{PA}=153^{\circ}\right)$.

We also find that $R 1$ and $R 2$ are not centered around the star (listed as $X\left(u_{x}\right)$ and $Y\left(u_{y}\right)$ offset in Table 2). The offset of the ellipse-centers with respect to the position of the stars means that the rings are either:

\section{- eccentric rings;}

1 Using the routine mpfitellipse.pro with the Interactive Data Language (IDL). 


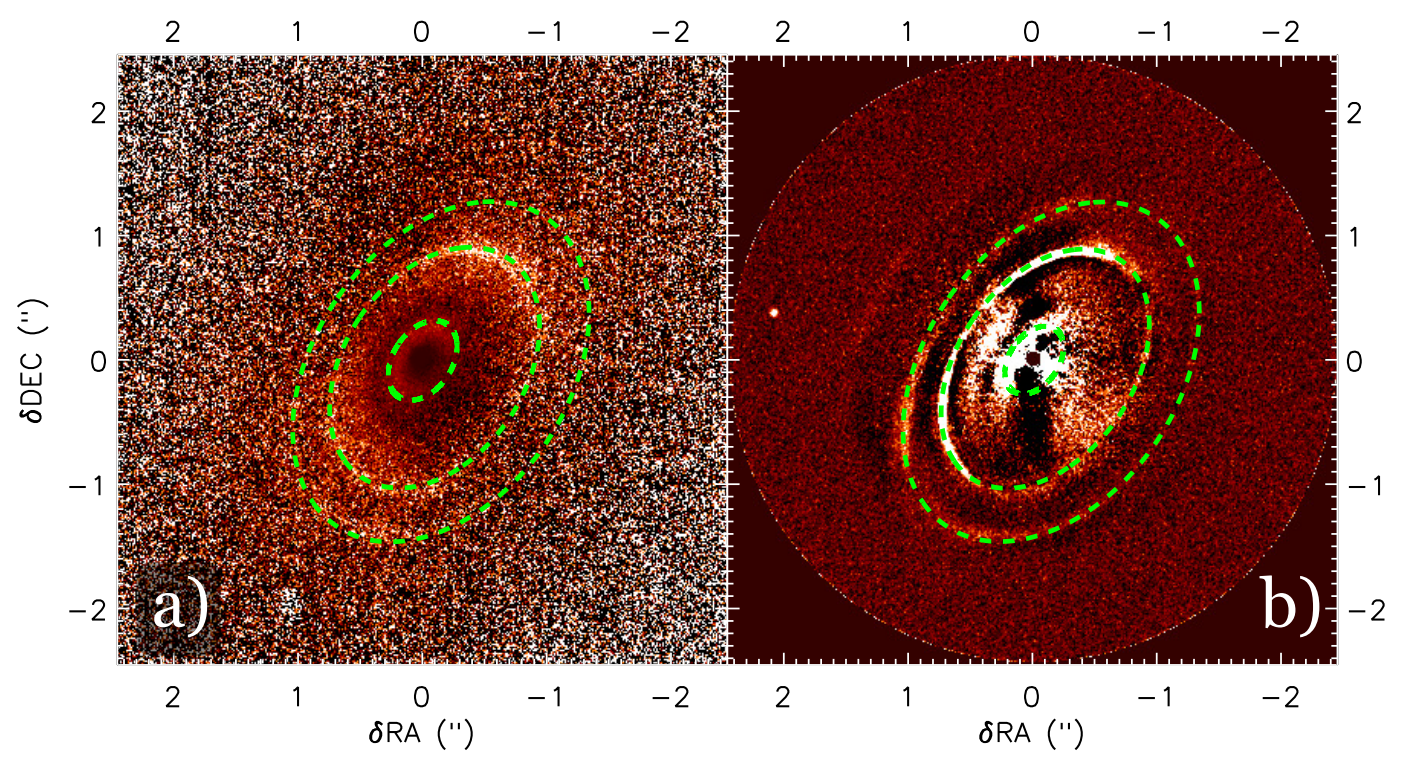

Fig. 5. a) IRDIS $J$-band PDI image after inclination-corrected $r^{2}$ scaling. b) IRDIS $H 23$-band TLOCI + ADI image. The overplotted green ellipses are the fits to $R 1, R 2$ and $I 1$, as listed in Table 2 .

Table 2. Ellipse parameters for the fits to the features listed in Figs. 5a and $\mathrm{b}$.

\begin{tabular}{|c|c|c|c|c|c|}
\hline & Parameter & ADI- $H 23$ & $\sigma_{H 23}$ & PDI- $J$ & $\sigma_{J}$ \\
\hline \multirow[t]{9}{*}{$R 1$} & Semi-major axis $\left({ }^{\prime \prime}\right)$ & 1.50 & 0.01 & 1.50 & 0.01 \\
\hline & Semi-minor axis ("') & 1.01 & 0.01 & 1.02 & 0.01 \\
\hline & RA offset $u_{x}\left({ }^{\prime \prime}\right)$ & -0.15 & 0.01 & -0.15 & 0.01 \\
\hline & Dec offset $u_{y}{ }^{\left({ }^{\prime \prime}\right)}$ & -0.10 & 0.01 & -0.10 & 0.01 \\
\hline & Offset angle $\left(^{\circ}\right)$ & 238 & 1 & 236 & 1 \\
\hline & $\mathrm{PA}\left({ }^{\circ}\right)$ & 145.7 & 1.0 & 144.2 & 0.8 \\
\hline & Inclination angle $\left(^{\circ}\right)$ & 47.3 & 1.0 & 47.0 & 0.8 \\
\hline & $H_{\tau=1}(\mathrm{au})$ & 44.9 & 2.2 & 44.7 & 1.7 \\
\hline & $H_{\tau=1} / r$ & 0.162 & 0.009 & 0.162 & 0.007 \\
\hline \multirow[t]{9}{*}{$R 2$} & Semi-major axis $\left({ }^{\prime \prime}\right)$ & 1.06 & 0.01 & 1.06 & 0.01 \\
\hline & Semi-minor axis ("') & 0.70 & 0.01 & 0.72 & 0.01 \\
\hline & RA offset $u_{x}\left({ }^{\prime \prime}\right)$ & -0.10 & 0.01 & -0.10 & 0.01 \\
\hline & Dec offset $u_{y}\left({ }^{\prime \prime}\right)$ & -0.07 & 0.01 & -0.06 & 0.01 \\
\hline & Offset angle $\left(^{\circ}\right)$ & 235 & 1 & 236 & 2 \\
\hline & $\mathrm{PA}\left({ }^{\circ}\right)$ & 145.4 & 1.3 & 144.3 & 1.4 \\
\hline & Inclination angle $\left({ }^{\circ}\right)$ & 48.5 & 1.3 & 46.8 & 1.4 \\
\hline & $H_{\tau=1}(\mathrm{au})$ & 30.9 & 2.4 & 29.6 & 2.2 \\
\hline & $H_{\tau=1} / r$ & 0.158 & 0.014 & 0.152 & 0.013 \\
\hline \multirow[t]{8}{*}{$I 1$} & Semi-major axis $\left({ }^{\prime \prime}\right)$ & 0.30 & 0.01 & 0.35 & 0.01 \\
\hline & Semi-minor axis $\left({ }^{\prime \prime}\right)$ & 0.20 & 0.01 & 0.24 & 0.01 \\
\hline & RA offset $u_{x}\left({ }^{\prime \prime}\right)$ & -0.01 & 0.01 & 0.00 & 0.01 \\
\hline & Dec offset $u_{y}\left({ }^{\prime \prime}\right)$ & 0.00 & 0.01 & 0.00 & 0.01 \\
\hline & Offset angle* $\left(^{\circ}\right)$ & 261 & & 209 & \\
\hline & $\mathrm{PA}\left({ }^{\circ}\right)$ & 145.5 & 4.2 & 144.5 & 4.3 \\
\hline & Inclination angle $\left({ }^{\circ}\right)$ & 49.0 & 3.9 & 47.7 & 4.1 \\
\hline & $H_{\tau=1} *(\mathrm{au})$ & 3.5 & & 1.4 & \\
\hline
\end{tabular}

Notes. ${ }^{(*)}$ For $I 1$, the errors on the ellipse offset $(u)$ are larger than the measured value. We therefore do not consider the value for the ellipse offset angle and $H_{\tau=1}$ to be significant.

- concentric circular disk components with considerable radially increasing thickness, as described by the height of the $\tau=1$ surface $\left(H_{\tau=1}(r)\right)$ above the disk midplane;

- an intermediate of the two extremes: an eccentric and a thick ring.
The directions in which $R 1$ and $R 2$ are offset from the star (listed as "Offset angle" in Table 2) are $\sim \mathrm{PA}+90$, meaning along the minor axes of the ellipses. These apparent ellipse displacements along the minor axes is a necessary condition for the offsets to be caused by a projection of the $\tau=1$ surface on the midplane due to the inclination of the system. Below, we explore the scenario of thick circular rings viewed at an inclination $i$ away from face-on. In this scenario, the disk in the direction opposite to the ellipse offsets (i.e., the eastern side, PA $\sim 56^{\circ}$ ) forms the near side. Without trying to explain the surface density distribution of the disk, in Fig. 6 we portray such a disk with thick rings. From the sketch we can derive that for any given ellipse with its center offset from the star-center with distance $u=\left(u_{x}^{2}+u_{y}^{2}\right)^{1 / 2}$, we can determine the height of the scattering surface $\left(H_{\tau=1}(r)\right)$ of this ring according to:

$\frac{u}{b}=\frac{H_{\tau=1}(r)}{r} \times \frac{\sin i}{\cos i}=\tan d \times \tan i$,

$H_{\tau=1}(r)=\frac{u}{b} \times \frac{r}{\tan i}=\frac{u}{\sin i}$.

In a similar fashion, Lagage et al. (2006) have determined the height of the disk around HD 97048 based on PAH emission maps. The main difference between the method used by Lagage et al. (2006) and our method is that the former have used isophotes, while we can use the sharp rings in the disk of RX J1615. It is clearly visible in Fig. 5 that the surface brightness of the rings is strongly varying with azimuth angle. To accurately determine $H_{\tau=1}$ based on isophotes it is necessary to correct for any azimuthal surface brightness variation, which requires radiative transfer modeling. To the best of our knowledge, this is the first example where $H_{\tau=1}$ can be determined strictly from geometrical constraints (i.e., model independent) in scattered light images.

\section{Polarized intensity profile}

Figure 7 shows the intensity profile of the $r^{2}$ scaled $Q_{\phi}$ image in $J$-band (Fig. 1c), after smoothing with four pixels. The profile is measured along PA $=146.5^{\circ}$, centered on the star (i.e., parallel to but not on the major axes of $R 1$ and $R 2$ ). The errors 


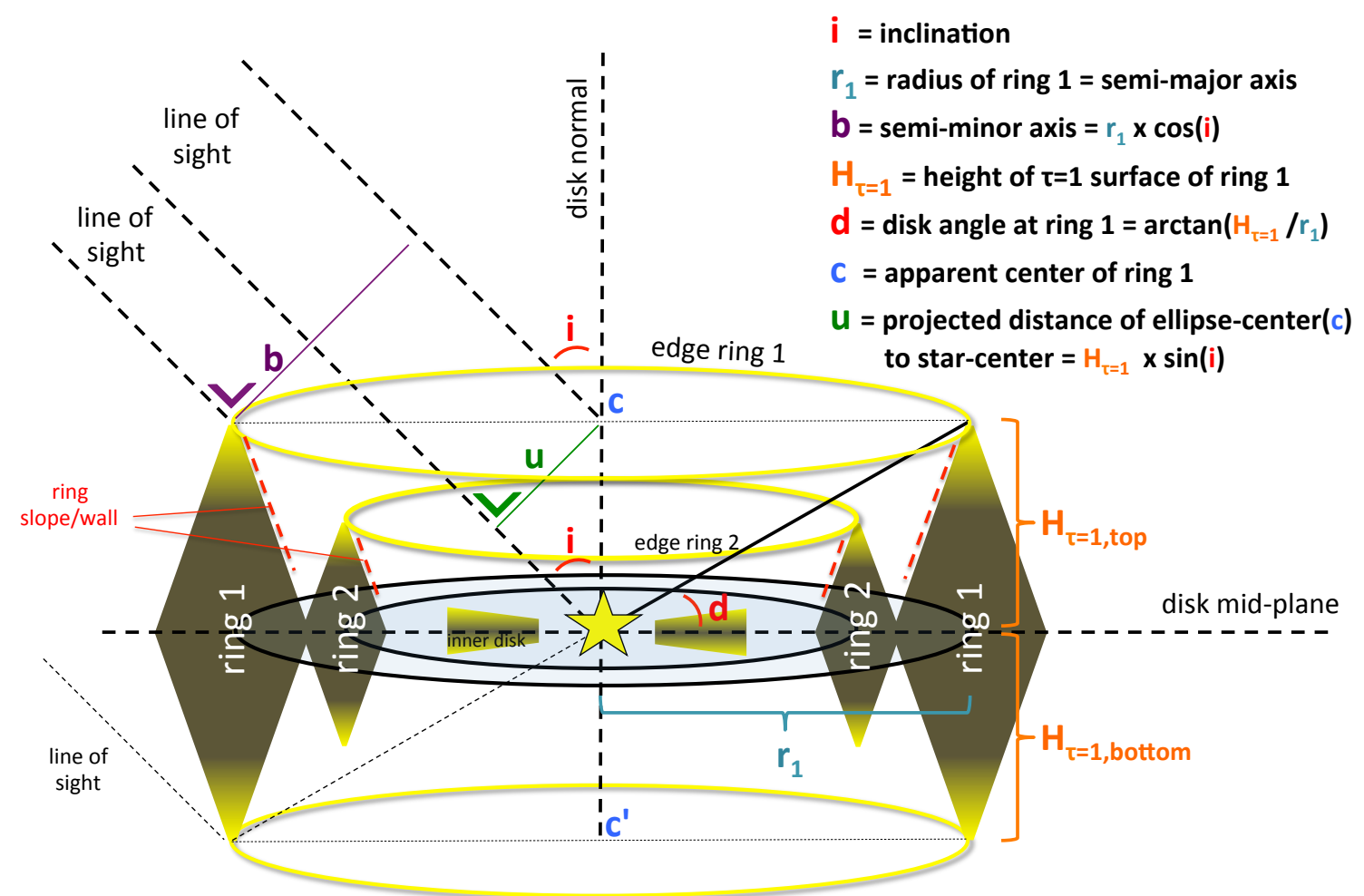

Fig. 6. Schematic view of a double ringed thick disk. Observed at inclination $i$, circular rings will appear elliptical. The height of the scattering surface of Ring $1\left(H_{\tau=1}(R 1)\right)$ can be found by determining the semi-minor axis $(b), i$ and the distance between the star and the center of the ellipse $(u)$. Note that this figure has the purpose of explaining how to determine $H_{\tau=1}$ for the different rings, not to portray ideas about the radial density distribution.

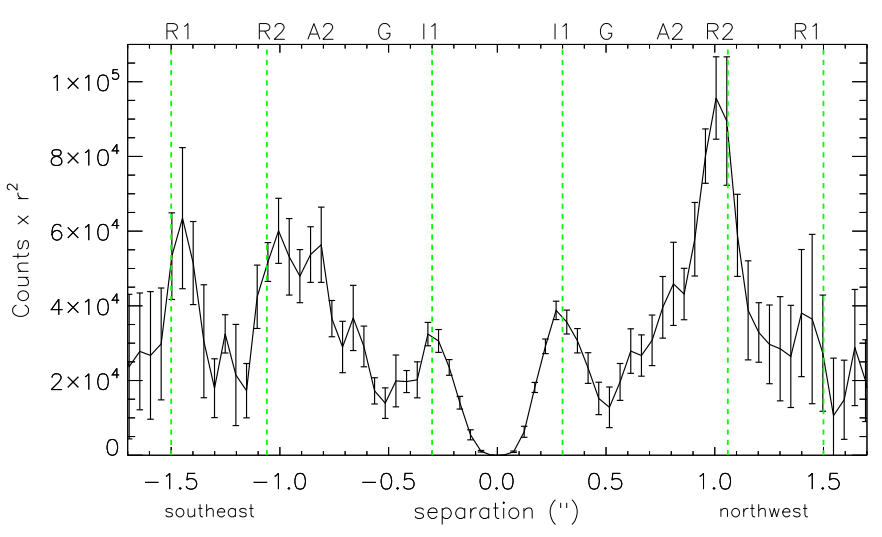

Fig. 7. $Q_{\phi}$ intensity profile of $r^{2}$ scaled $J$-band image (Fig. 1c) along $\mathrm{PA}=146.5^{\circ}$. The green dashed lines indicate the semi-major axes of the ellipses $R 1, R 2$ and $I 1$. We note that although this profile is measured for a PA similar to those of the ellipses, the profile does include the star center, and therefore does not lie on the major axes of $R 1$ and $R 2$. This explains why the peaks of the rings lie at closer separations than their semi-major axes.

are the standard deviation measured over the same aperture in the $U_{\phi}$ image (Fig. 1f), divided by the square root of the number of pixels. The disk features which can be distinguished in the profile are listed along the top axis of the plot. Due to the ellipse-center offset with respect to the star, the profile does not cross the ansae of the ellipses, which becomes visible when we compare the position of peaks in the intensity profile with the semi-major axis of the ellipses for $R 1$ and $R 2$, annotated with green dashed lines (values adopted from Table 2). Although the feature is not clearly visible in Fig. 1c, we can recognize the two peaks of $A 2$ in the $Q_{\phi}$ intensity profile. The gap $G$ is visible at $r \sim 0.5^{\prime \prime} \approx 93 \mathrm{au}$. van der Marel et al. (2015) find a tentative dust gap between $110-130 \mathrm{au}\left(0.6^{\prime \prime}-0.7^{\prime \prime}\right)$ in the $690 \mathrm{GHz}$ continuum profile. This sub-mm gap lies outside the gap we detect in the $J$-band data.

\subsection{Point sources}

Four point sources have been detected in the NACO $K_{\mathrm{s}}$-band image, and are indicated in Fig. 4a. In the IRDIS data (Fig. 4b), we detect no point sources within the ringed disk structure, out to $A 1$. We do detect nine point sources outside the outermost disk component $A 1$, including all four point sources detected with NACO. The five new companion candidates are highlighted with white boxes in Fig. 4b. For reference, we included the white boxes at the same locations in Fig. 4a, even though these point sources are not detected by NACO. The astrometry and photometry of the six innermost candidates detected with IRDIS were derived using the LAM-ADI pipeline (Vigan et al. 2012, 2016) using injection of fake negative planets in the pre-processed ADI data cubes. The position and flux of the negative fake companion are adjusted using a Levenberg-Marquardt least-squares minimisation routine where we try to minimise the residual noise after ADI processing in a circular aperture of radius $\lambda / D$ centered on the position of the companion candidate. The error bars for the fitting process are then calculated by varying the position and contrast of the fake companion until the variation of the reduced $\chi^{2}$ reaches a level of $1 \sigma$. For the speckle subtraction, we have used a PCA analysis (Soummer et al. 2012) where five modes are subtracted. At the distances of the outer three candidates there is negligible speckle noise negating the need for such postprocessing. For these we perform basic relative photometry after 

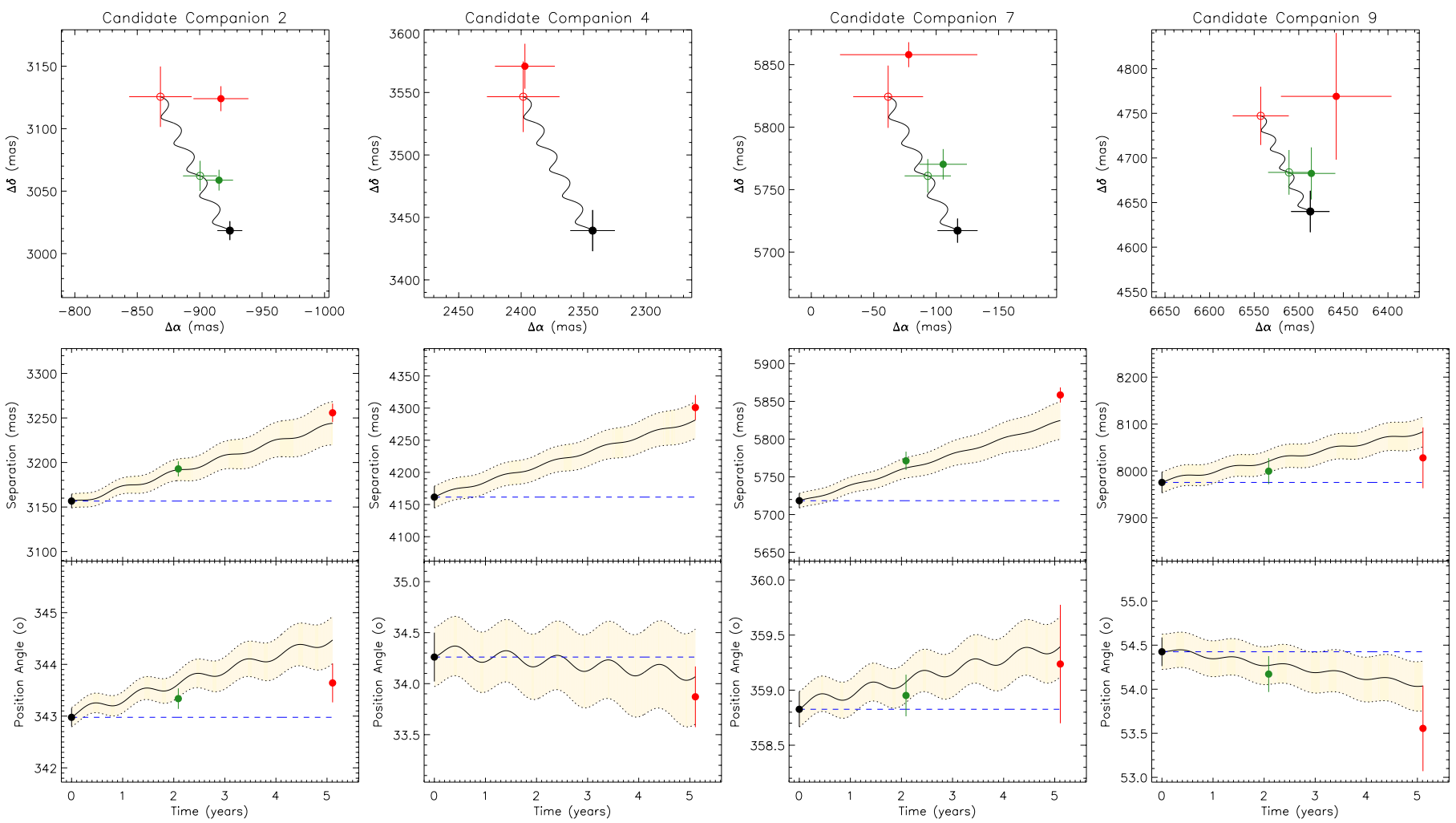

Fig. 8. Astrometry for the four point sources detected (solid points) by both NACO (black and green) and IRDIS (red) and the expected values for background objects (open points). Data points are color coded by date, not by instrument. These point sources are highlighted with the green squares in Fig. 4. The numbers of the candidate companions listed in the title of each panel column are the same as shown in Fig. 4. All four point sources are shown to not be co-moving with RXJ1615.

subtracting a radial profile and stacking the de-rotated frames. The astrometry and photometry measurements for the companion candidates are reported in Table 3. For IRDIS, we have adopted a true north direction of $-1.81 \pm 0.30^{\circ}$ and a plate scale of $12.27 \pm 0.016 \mathrm{mas} /$ pix, based on astrometric calibrations performed on the theta Ori B field (Maire et al. 2016; Vigan, priv. comm.). For the NACO observations of 2010, we adopted a truenorth of $0.67 \pm 0.13^{\circ}$ and a plate scale of $13.231 \pm 0.020$ mas; for 2012 we used a true-north of $0.65 \pm 0.14^{\circ}$ and a plate scale of $13.234 \pm 0.021$ mas. The final error bars for the photometry include the fitting error detailed above; the uncertainties on the star center (1 mas); and the level of noise residuals estimated at the same separation as the detections.

In Fig. 8 we compare the astrometry for the four point sources detected with NACO with that of our IRDIS detection. We determine them to not be co-moving, and therefore not associated to the RXJ1615 system. The five remaining candidates, seen in Fig. 4b, require follow-up observations to determine whether they are bound to RX J1615.

No significant point-source signals were found in any of the Keck NIRC2 SAM datasets, and so we place limits on their detectability by drawing 10000 simulated closure phase datasets consistent with Gaussian random noise using the measured uncertainties. For each combination of separation, contrast and PA on a $3 \mathrm{D}$ grid, the simulated datasets were compared to a binary model. The $(3.3 \sigma)$ detection limits were calculated as the point at which the binary model gave a worse fit to $99.9 \%$ of the simulated datasets. The datasets probe separation ranges as small as 30 mas, and achieve contrast limits that are approximately flat at larger separations. The 2014-06-10 data allow us to rule out objects with $\Delta K^{\prime}<5.4 \mathrm{mag}\left(>20 M_{\text {jup }}\right.$ for a system age of
Table 3. Astrometry and photometry for nine companion candidates relative to RX J1615.

\begin{tabular}{c|cccccc}
\hline \hline Obj & Date & Instr. & Filt. & $\Delta$ RA (mas) & $\Delta$ Dec (mas) & $\Delta$ mag \\
\hline cc1 & $15 / 05 / 2015$ & IRDIS & $H 2$ & $2086 \pm 16$ & $405 \pm 25$ & $13.14 \pm 0.42$ \\
& $15 / 05 / 2015$ & IRDIS & $H 3$ & $2090 \pm 16$ & $397 \pm 25$ & $13.17 \pm 0.34$ \\
\cline { 2 - 7 } cc & $05 / 04 / 2010$ & NACO & $K \mathrm{~s}$ & $-924 \pm 10$ & $3018 \pm 8$ & $8.47 \pm 0.12$ \\
& $07 / 05 / 2012$ & NACO & $K \mathrm{~s}$ & $-916 \pm 11$ & $3059 \pm 8$ & $8.48 \pm 0.13$ \\
& $15 / 05 / 2015$ & IRDIS & $H 2$ & $-907 \pm 22$ & $3242 \pm 9$ & $8.37 \pm 0.11$ \\
& $15 / 05 / 2015$ & IRDIS & $H 3$ & $-909 \pm 22$ & $3243 \pm 10$ & $8.40 \pm 0.10$ \\
\cline { 2 - 7 } cc3 & $15 / 05 / 2015$ & IRDIS & $H 2$ & $2722 \pm 27$ & $1854 \pm 33$ & $12.90 \pm 0.35$ \\
& $15 / 05 / 2015$ & IRDIS & $H 3$ & $2722 \pm 27$ & $1859 \pm 33$ & $12.80 \pm 0.65$ \\
\cline { 2 - 7 } cc4 & $05 / 04 / 2010$ & NACO & $K \mathrm{~s}$ & $2343 \pm 18$ & $3439 \pm 17$ & $10.45 \pm 0.31$ \\
& $15 / 05 / 2015$ & IRDIS & $H 2$ & $2397 \pm 24$ & $3571 \pm 18$ & $10.67 \pm 0.08$ \\
& $15 / 05 / 2015$ & IRDIS & $H 3$ & $2397 \pm 24$ & $3571 \pm 18$ & $10.70 \pm 0.08$ \\
\cline { 2 - 7 } cc5 & $15 / 05 / 2015$ & IRDIS & $H 2$ & $-8 \pm 27$ & $-3745 \pm 5$ & $12.20 \pm 0.17$ \\
& $15 / 05 / 2015$ & IRDIS & $H 3$ & $-8 \pm 27$ & $-3744 \pm 5$ & $12.19 \pm 0.17$ \\
\cline { 2 - 7 } cc6 & $15 / 05 / 2015$ & IRDIS & $H 2$ & $242 \pm 39$ & $4728 \pm 9$ & $12.30 \pm 0.22$ \\
& $15 / 05 / 2015$ & IRDIS & $H 3$ & $234 \pm 39$ & $4730 \pm 9$ & $12.26 \pm 0.18$ \\
\cline { 2 - 7 } cc7 & $05 / 04 / 2010$ & NACO & $K \mathrm{~s}$ & $-117 \pm 16$ & $5717 \pm 10$ & $9.88 \pm 0.24$ \\
& $07 / 05 / 2012$ & NACO & $K \mathrm{~s}$ & $-106 \pm 19$ & $5770 \pm 12$ & $9.96 \pm 0.27$ \\
& $15 / 05 / 2015$ & IRDIS & $H 2$ & $-78 \pm 55$ & $5858 \pm 10$ & $9.74 \pm 0.39$ \\
& $15 / 05 / 2015$ & IRDIS & $H 3$ & $-78 \pm 55$ & $5857 \pm 10$ & $9.71 \pm 0.38$ \\
\cline { 2 - 7 } cc & $15 / 05 / 2015$ & IRDIS & $H 2$ & $3065 \pm 46$ & $-5007 \pm 36$ & $11.45 \pm 0.43$ \\
& $15 / 05 / 2015$ & IRDIS & $H 3$ & $3059 \pm 46$ & $-5010 \pm 36$ & $11.29 \pm 0.40$ \\
\cline { 2 - 6 } cc9 & $05 / 04 / 2010$ & NACO & $K \mathrm{~S}$ & $6487 \pm 22$ & $4640 \pm 23$ & $9.11 \pm 0.16$ \\
& $07 / 05 / 2012$ & NACO & $K \mathrm{~s}$ & $6486 \pm 27$ & $4683 \pm 29$ & $9.26 \pm 0.19$ \\
& $15 / 05 / 2015$ & IRDIS & $H 2$ & $6458 \pm 62$ & $4769 \pm 71$ & $10.65 \pm 0.46$ \\
& $15 / 05 / 2015$ & IRDIS & $H 3$ & $6439 \pm 87$ & $4790 \pm 95$ & $10.75 \pm 0.42$ \\
\hline
\end{tabular}

1.4 Myr), while the 2012-07-08 and 2012-04-14 data reach similar contrasts of $5.0 \mathrm{mag}$ and $5.2 \mathrm{mag}$, respectively. 


\section{Discussion}

\subsection{Disk geometry}

The explanation for the apparent ellipse offsets $u$ by the projection of the $\tau=1$ surface at height $H_{\tau=1}$ above the midplane, as given in Sect. 4.1, implies that the northeast $(\mathrm{PA} \sim 56)$ is the near side of the disk (i.e., pointing towards earth). Min et al. (2012) and Dong et al. (2016) show that the predominance of forward scattering in the near side of disks gives it, in general, a larger surface brightness in total intensity than the far side. The ADI intensity image of Fig. 2 shows the northeastern sides of $R 1, R 2$ and $I 1$ to be brighter than their southwestern sides, confirming that the northeast is the near side of the disk.

In Sect. 4.1, we find the disk inclination $i=47 \pm 2^{\circ}$, which is in good agreement with both the disk inclination derived by van der Marel et al. (2015) and the inclination of the stellar rotation axis derived in Appendix A from $v \sin i=13.0 \mathrm{~km} \mathrm{~s}^{-1}$ (Wichmann et al. 1999; where the stellar rotational velocity $v$ depends on the determination of the stellar radius from its luminosity, which in turn depends on the interstellar extinction $A_{V}=0$ mag, as suggested by Manara et al. 2014). Higher extinction (e.g., $A_{V}=0.4$ mag Andrews et al. 2011) would lead to a lower inclination of the stellar rotation axis, which does not agree with the inclination of the disk. However, the disk inclination would be altered if the observed ellipses do not trace the rings' highest surfaces above the midplane (called "edge ring $1 / 2$ " in Fig. 6). Without advanced radiative transfer modeling it is not possible to differentiate in our scattered light images between starlight scattered off the ring edges and light scattered off the slopes/walls of the rings (called "ring slope/wall" in Fig. 6). Consequently, the ring edges might truly lie further out than our ellipse fits. This effect would not be symmetric; we are more likely to have a larger contribution of the wall in the backward scattering side (southwest, near the minor axis) of the rings. Therefore, if the apparent ellipses are affected by scattering by the ring walls, the ellipticity of the true ring edges (and their inclination) will be slightly lower and the ring offsets larger.

\subsubsection{Nature of the ring structures}

It is tempting to interpret the rings and arcs detected in the disk as spatial variations in surface density. However, our NIR scattered light detections of the disk trace the disk surface for the micronsized dust grains. We cannot unambiguously determine whether the rings are either a manifestation of variations in the scale height caused by spatial variations in temperature (e.g., due to shocks), or variations in the surface density either caused by dead zones or by massive planets carving a gap in the gas surface density. Dust trapping by local peaks in the gas pressure will appear different for small and large dust grains. Pinilla et al. (2016) show that if we can measure a difference between the density enhancements for the different grain sizes, we can discriminate between dead zones creating a bump in the gas pressure and massive planets carving a gap in the gas disk. Furthermore, the mass of a gap carving planet can be predicted by the amount of the displacement between large grain and small grain peaks in the surface density for a given gas viscosity (de Juan Ovelar et al 2016). Large baseline sub-mm (ALMA) observations are therefore required to study the origin of the ring structures. A resolution comparable to our SPHERE observations will be needed in order to resolve the ring structure (when present for large grains), and accurately compare their radius with those of the rings in the surface of the small grain dust disk presented in this study.
Another asymmetry is detected in the polarized intensity profile of Fig. 7, which roughly traces the major axes of the ellipses. The peaks of $I 1$ and $R 2$ are brighter in the northwest than their southeastern counterparts, while the opposite (brighter in the southeast) is true for $A 2$ and $R 1$. The fact that these brightness asymmetries along the rings are oscillating between northeast and southwest can possibly be explained with shadowing: the brighter parts of the rings might have a larger scale height than their fainter counterparts. If each ring outside of $I 1$ is just marginally rising out from the shadow of the ring directly inside, this would cause a brighter ring segment in the inner ring to cast a larger shadow on the outer ring, hindering the stellar radiation to heat up the segment in the outer ring with similar azimuth angle. The opposite happens for the faint segments, which have a smaller scale height, casting less of a shadow on the next ring: this next ring receives more stellar radiation, heating it up and allowing it to "puff up" more, making it brighter than the opposite side of the same ring. Shadowing of the outer rings by the inner rings is only possible when the flaring of the disk is very small. When we compare the disk angle (parameter $d$ in Fig. 6) for both rings, we find that $\tan d(R 1)=H_{\tau=1} / r(R 1) \approx 0.16$ is marginally larger than $\tan d(R 2)=H_{\tau=1} / r(R 2) \approx 0.15$, which confirms that the disk flaring is minimal.

Although ALMA is detecting an increasing number of protoplanetary disks with multiple rings (e.g. HL Tau), very few have been detected in scattered light. To the best of our knowledge, only TW Hya (Rapson et al. 2015; van Boekel et al. 2016), HD 141569A (e.g. Weinberger et al. 1999; Perrot et al. 2016) and HD 97048 (Ginski et al. 2016) display multiple rings in scattered light. The inclination of HD 141569A (between $i=45^{\circ}$ and $51^{\circ}$ for the different rings, Biller et al. 2015; and $i=56^{\circ}$ for the entire disk, Mazoyer et al. 2016) is comparable to RX J1615. The ringed structure also looks very similar to RX J1615, because the rings are relatively sharp compared to the larger radial extent of its gaps. As we discussed above, in a disk with low flaring (hereafter "flat", i.e. $H / r=$ const.), a small ripple in the disk surface can cast large shadows outward. Indeed, Thi et al. (2014) suggest that the disk of HD 141569A is very flat, while the radial extent of rings in the surface of more flaring disks, such as HD 97048 and TW Hya, is of similar size or larger than the width of their gaps. We therefore suggest that the sharpness of rings in the surface of a primordial disk can be used as a tracer for the degree of flaring of the disk surface. This argument is based on the assumption that the apparent gaps are (mainly) due to shadows cast by ripples in the scattering surface, rather than true gaps in the surface density of the disk. To test this hypothesis we would need better knowledge of the scale height of the disk (e.g., through high angular resolution gas observations with ALMA) in several disks that show multiple ring structures in scattered light.

\subsubsection{A1: additional ring or bottom of $R 1$}

For the arc-like structure $(A 1)$ in Fig. 2, we consider two explanations to be equally plausible: it could either be an additional ring, at a separation from the star $(r)$ larger than for $R 1$. However, the similarity of this arc to the shape of $R 1$ at similar PAs $\left(10^{\circ} \lesssim \mathrm{PA} \lesssim 100^{\circ}\right)$ is much stronger than when we compare $R 1$ and $R 2$ at these PAs. Therefore, we suggest an alternative explanation for $A 1$ as it being the backward facing end of $R 1$, comparable to the bottom part of ring 1 in Fig. 6.

To speculate on the shape of the ellipse for either explanation of $A 1$, we added two ellipses (red and purple) to Fig. 9b. These ellipses (explained in the cartoons of Figs. 5c and d) are fixed at the intersection between $A 1$ and the minor axis of the 
J. de Boer et al.: SPHERE imaging of the disk and companion candidates around RX J1615

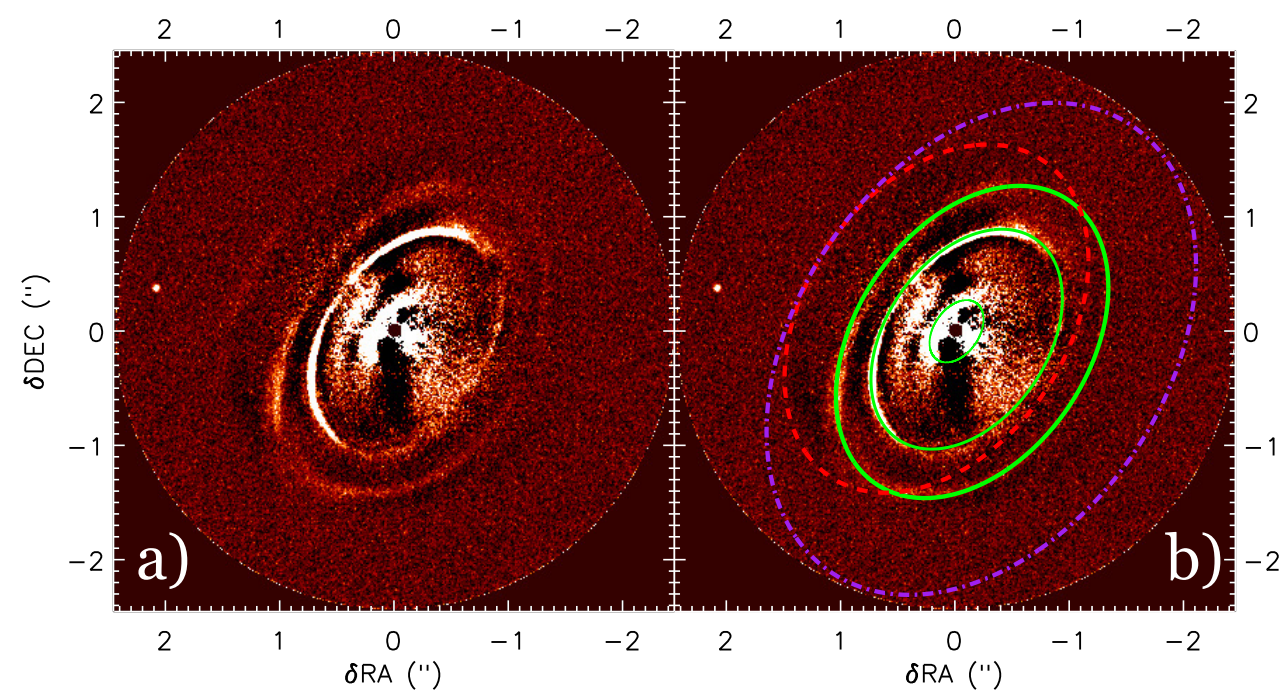

c)

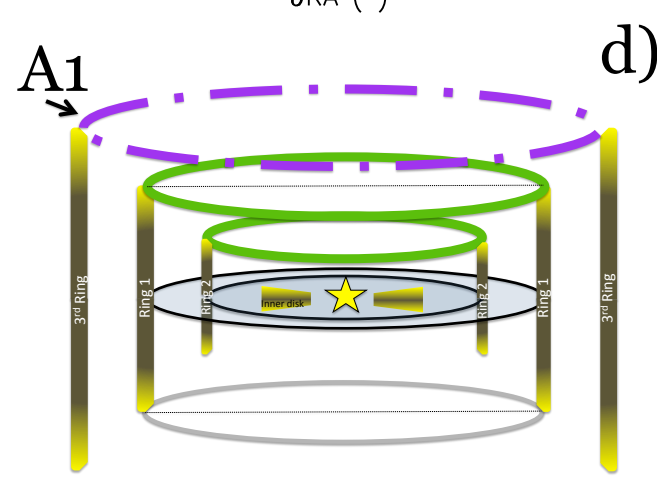

Fig. 9. b) IRDIS H23-band TLOCI + ADI image. The overplotted green ellipses (solid lines) are the fits to $R 1, R 2$ and $I 1$, as listed in Table 2 , while the red (dashed) and purple (dash dot) ellipses depict the two scenarios where $A 1$ is either the backward-facing side of ring 1 , or the forward-facing side of a 3rd ring further out (sketched in panels c) and d)), respectively. c) First explanation for $A 1$, for the same considerations as Fig. 6: $A 1$ is the bottom/backward facing side of Ring 1 , for which $R 1$ is the top/forward facing side, (i.e., $r(A 1) \approx r(R 1)$ ). d) Second explanation for $A 1$ : $A 1$ is the top/forward facing side of a third ring, with $r(A 1)>r(R 1)$. Both the red and the purple ellipses are selected based on the assumption that compared to $R 1$ and $R 2, A 1$ would have roughly the same aspect ratio between major and minor axes as well as the same ratio between ellipse-star offset and the major axis.

disk; have the same $P A$ as $R 2$, and the same ratio of major/minor axes (i.e., inclination). The final constraints we used for the two ellipses are: firstly that the ring has either a similar (or larger) $H_{\tau=1}$ for the scenario where $A 1$ is the backward facing side of $R 1$ (red ellipse in Figs. $9 \mathrm{~b}$ and c), and secondly that $H_{\tau=1}$ of a new ring further out (purple ellipse in Figs. $9 \mathrm{~b}$ and d) needs to be large enough to rise from the shadow of $R 1$. As we discussed in Sect. 5.1, this means that $H_{\tau=1} / r(A 1)>H_{\tau=1} / r(R 1)$. From Eq. (10) and the constraints above we derive that the absolute value of the ellipse $(x-y)$ offset $(|u|)$ divided by the minor axis (b) for $A 1$ should be:

$|u| / b(A 1) \geq|u| / b(R 1)=0.18$.

We have used $|u| / b(A 1)=0.18$ for both ellipses in Fig. 9b, which can be considered as a best guess for the red ellipse and a lower limit for the purple ellipse: larger purple ellipses (which will have larger $|u| / b$ ) are not ruled out. Deeper observations of this structure will reveal which of the two scenarios is true: detecting a larger (azimuth coverage of the) ring segment will enable us to distinguish between the red and the purple ellipse scenarios.

In order to fix the red ellipse on the intersection between the minor axis and the detected $A 1$ feature, both a larger semi-major axis and $x$ and $y$ offset (in opposite direction) are needed for the red ellipse (see Table 4) than what we found for the green
Table 4. Ellipse parameters for feature $A 1$ in Fig. 2c.

\begin{tabular}{c|lll}
\hline \hline & Parameter & Red & Purple \\
\hline$A 1$ & Semi-major axis $\left(^{\prime \prime}\right)$ & 1.66 & 2.35 \\
(no fit) & Semi-minor axis (") & 1.14 & 1.61 \\
& RA offset $u_{x}\left({ }^{\prime \prime}\right)$ & 0.16 & -0.23 \\
& Dec offset $u_{y}\left({ }^{\prime \prime}\right)$ & 0.11 & -0.16 \\
& Offset angle $\left(^{\circ}\right)$ & 56 & 236 \\
& $H_{\tau=1}(\mathrm{au})$ & 49.2 & 70.7 \\
& $H_{\tau=1} / r$ & 0.16 & 0.16 \\
\hline
\end{tabular}

Notes. In Fig. 5, the red ellipse shows the scenario where $A 1$ is the bottom side of $R 1$; the purple ellipse shows $A 1$ as a separate outermost ellipse.

ellipse of $R 1$ in Table 2 . This either hints at a structure with $H_{\tau=1 \text {, bottom }}>H_{\tau=1 \text {,top }}$ or $r(A 1)>r(R 1)$. The scattering-angle for the backward facing part of ring 1 will be different than the forward facing part: moving the beam back into denser regions of the disk instead of away from higher density (as for the forward facing $R 1$ ). This will bring the backward facing $\tau=1$ surface to lie further from the midplane than for the forward facing surface at the same distance $r$. 


\subsection{Possible disk sculpting companion}

Should the ring $A 1$ turn out to be the backward facing side of ring 1, as we illustrate in Fig. 9c, we would expect there to be a fairly massive planetary companion to the system. To make the backward facing side of ring 1 visible, the disk needs to be truncated at most $H(A 1) \times \tan i=54$ au beyond ring 1 , which lies at $1.66 \times 185=307$ au (where we used the semi-major axis of $1.66^{\prime \prime}$ for $A 1$, instead of the $1.50^{\prime \prime}$ for $R 1$, since the former will be dominated by the outer edge, the latter by the inner edge of ring 1). The disk truncation at $r \approx 361$ au can be a consequence of a massive planet beyond this radius, opening a gap in the disk. Detection of such a companion should be possible with our IRDIS ADI observations as long as the planet signal is not obscured by the disk (e.g., when the planet is on the western side of its orbit, or the truncation is not performed by multiple lower mass planets). Should companion candidate 1 (cc1, see Fig. 2) be associated with the system, it could provide the required disk truncation.

To determine the mass of cc1 (if it is indeed bound to the system) we first need to find the age of the system. In Appendix A we determine the rotation period $\left(P_{\star}=5.72 \pm 0.01 \mathrm{~d}\right)$, spectral type $(\mathrm{K} 5-\mathrm{K} 6)$ and temperature $\left(T_{\text {eff }}=4100 \pm 100 \mathrm{~K}\right)$ of RX J1615, which we use to determine the inclination of the stellar rotation axis and constrain the stellar age and mass. Overall, the properties of the star such as accretion, characteristics of the disk, the kinematic, and the limits from lithium and rotation period indicate an age less than $5 \mathrm{Myr}$. Following the same process as Wahhaj et al. (2010) we use the models of Siess et al. (2000) to determine the age of RXJ1615 from isochrones. Adopting the luminosity $L=1.07 \pm 0.11 L_{\odot}$ and effective temperature $T_{\text {eff }}=4100 \pm 100$, as stated before, we find age range of $1.8 \pm 0.6 \mathrm{Myr}$ and a stellar mass of $0.8 \pm 0.1 M_{\odot}$.

Using the COND model (Baraffe et al. 2003), our observed $H$-band magnitude, and the assumed age of the system, we estimate the mass of $\mathrm{cc} 1$ to be $0.8 \pm 0.1 M_{\text {jup }}$. In this estimation, we have assumed the signal to be dominated by thermal emission of the planet, rather than the emission by a circumplanetary accretion disk. If we assume that cc1 is a companion orbiting RX J1615 along the midplane of the disk, its deprojected distance to the star would be $\sim 540$ au. Assuming that the maximum gap size created by a companion on a circular orbit is 5 Hill Radii (Dodson-Robinson \& Salyk 2011; Pinilla et al. 2012), at this separation we would need a planet with higher mass $\left(\gtrsim 1.5 M_{\text {jup }}\right)$ to fully truncate the gas disk at $r=361 \mathrm{au}$, or the planet needs to have an elliptical orbit. However, if the gas density is sufficiently reduced in the outer disk, in particular at the planet gap, the $\mu \mathrm{m}$ sized grains can become decoupled to start drifting inwards, leading to a smaller disk truncation of the micron-sized particles compared to the gas. Without the presence of a planetary gap, the micron sized particles would remain coupled and the truncation in scattered NIR light would be further out than observed. Whether the potential drift of the $\mu \mathrm{m}$ grains in a continuous gas disk suffices to reduce the disk optical depth enough to enable the transmission of light scattered by the backward-facing side of ring 1 requires radiative transfer modeling, which is beyond the scope of this study.

We show in Fig. 10 the locations of our candidate companions on a color magnitude diagram, and included previously known brown dwarfs and planetary companions for reference. Upon first inspection of this diagram, we might assume that cc1 is more likely to be a background object as it, along with all remaining candidates, has an $H 2-H 3$ color typical of a background $\mathrm{M}$ dwarf. However, we know that some planetary

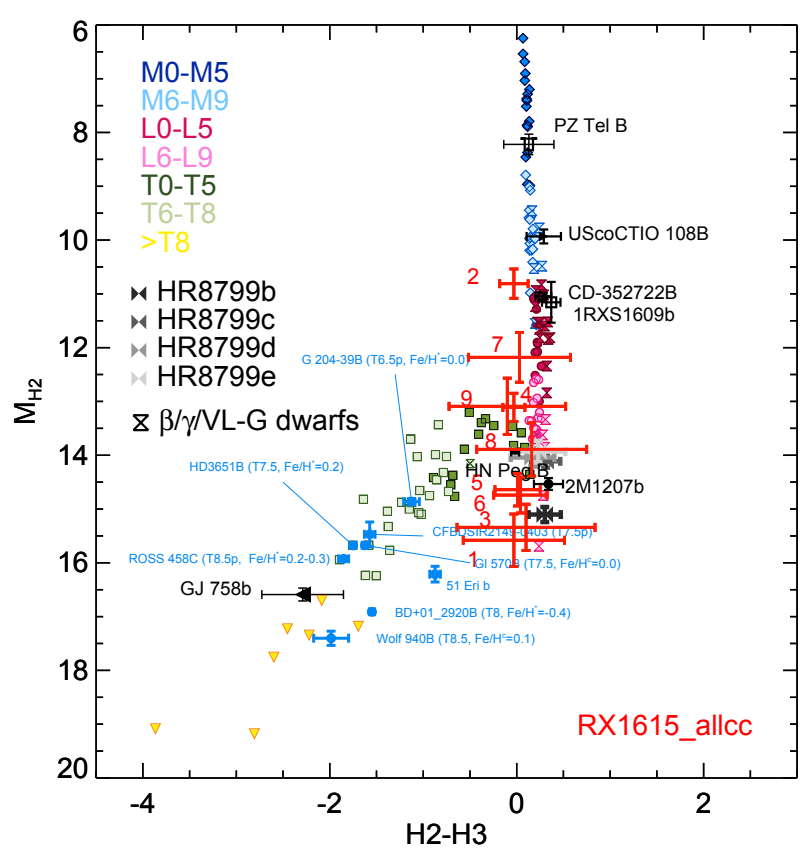

Fig. 10. Color magnitude diagram displaying our candidate companions compared to already known planetary companions and brown dwarfs. Candidate companions are displayed, and numbered, in red with corresponding error bars.

companions (such as those of HR8799 and 2M1707) do have colors within the range of $\mathrm{ccl}$ so we can claim nothing for certain. Indeed RXJ1615 is treading on new territory being so young and low mass. If cc1 is attributed to the RX J1615 system we would normally expect there to be a methane signature (which would yield a negative $H 2-H 3$ color). However, to date no observational evidence for methane emission in such young and low mass systems exists, leaving the possibility open that $\mathrm{cc} 1$ is bound to the RX J1615 system. Follow-up observations of cc1 are needed to confirm whether the candidate is co-moving with RX J1615. If bound cc1 would be an ideal target for characterization with the IRDIS low resolution long-slit spectroscopy mode (Vigan et al. 2008).

Besides the option of the disk being truncated by a single massive planet as an explanation of why $A 1$ could possibly be the backward facing side of $R 1$, multiple lower mass planets can provide a similar truncation of the disk. Such lower mass planets might remain undetectabel by SPHERE. For this reason we chose to include the contrast plot seen in Fig. 11, showing the contrast in two cuts across the center of RX J1615, parallel to the major and along minor axes of the rings. A conventional contrast curve, where the contrast is measured over concentric annuli would contain the disk signal in such a way that one can no longer tell if a peak is caused by a ring or by something else. However, outwards of R1 this is not an issue. We have inserted the measured signal of $\mathrm{cc} 1$ at its appropriate radial separation for reference.

\subsection{Wavelength dependent surface brightness of $R 2$}

$R 2$ is clearly detected in H23-band ADI image; it is fainter in the $J$-band PDI data; and can no longer be distinguished against the background disk signal in the $R^{\prime}$-band PDI image. We detect a similar wavelength dependence of the $R 2$ surface brightness when we compare the three wavelength regimes of the IFS in Fig. 3. If this wavelength dependence is truely astrophysical, 


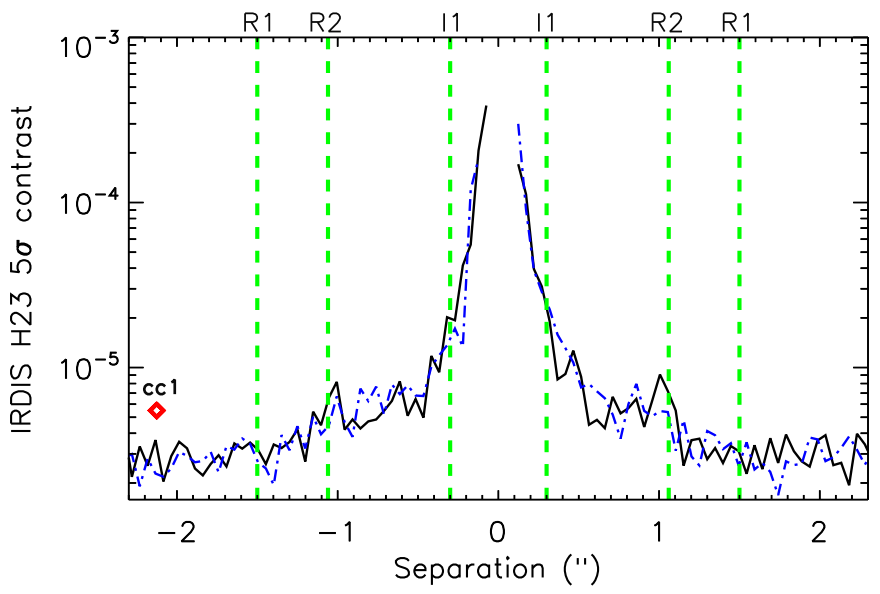

Fig. 11. $5 \sigma$ contrast measured in the H23 TLOCI reduction of May 15 2015 , along $\mathrm{PA}=146^{\circ}$, parallel to the ellipse's major axes (black solid line, negative is southeast); and PA $=56^{\circ}$, the ellipse's minor axes (blue dash-dotted line, negative is northeast). The vertical green dashed lines display the semi-major axes of $R 1, R 2$, and $I 1$ as annotated above the top axis of the plot. The red diamond shows the measured signal of cc1 (which, we find, corresponds to $0.8 \pm 0.1 M_{\text {jup }}$ ). Notice that the actual location of cc 1 does not coincide with either the minor or the major axes of the disk.

it could be indicative of spatial/radial variations in the chemistry or grain size distribution throughout the disk. However, the low Strehl ratio and FWHM of the ZIMPOL data described in Sect. 2 can also be responsible for washing out a structure which is not resolved in the radial direction, smoothing out the unresolved structure so much that we can no longer distinguish $R 2$ from the surface brightness of its surroundings. The simultaneous measurements at the three IFS wavelength regimes suffer from the same systematic effect: the Strehl ratio increases for longer wavelength.

Since disk signal is present both directly inside and outside of $R 2$, we cannot determine if the low resolution of the $R^{\prime}$ image has washed out the disk signal. Convolving a radiative transfer model with the PSFs of the different observations will be the best way to answer whether we are looking at a true astrophysical change with wavelength of the surface brightness or rather at systematic effects due to the difference in Strehl ratio and FWHM. However, creating a realistic radiative transfer model lies outside the scope of this paper and is left for future work.

\section{Conclusion}

We have studied the system of RXJ1615 with four observing modes of the high contrast imager VLT/SPHERE. We detect the disk of RXJ1615 in scattered light for the first time, from the optical $R^{\prime}$-band to the NIR $K_{\mathrm{s}}$-band, with all high contrast imaging modes used in this study. The $J$ and H23-band images show three elliptical disk components surrounding the star and two arc-like features. The two outer ellipses $R 1$ and $R 2$ have their centers offset from the position of the star. The simplest explanation for the elliptical features is that we see a disk at inclination $i=47 \pm 2^{\circ}$ which contains an inner disk $I 1$ surrounded by two circular rings, for which the height of the $\tau=1$ surface increases with its distance to the star. Ellipse fitting yields the major axes (i.e., radii ( $r$ ) of the rings) for $R 1$ to be $278 \pm 2 \mathrm{au}$, for $R 2 r=196 \pm 2 \mathrm{au}$, and for $I 1 r=56 \pm 2$ au. A tentative cavity is detected inside the inner disk. The apparent offsets between star and ring centers allow us to determine the height of the scattering surface above the midplane: $H_{\tau=1}(R 1)=44.8 \pm 2.3$ au and $H_{\tau=1}(R 2)=30.2 \pm 2.4$ au.

For the outermost disk feature $A 1$, our detection was not deep enough to determine its origin. Deeper observations are needed to determine whether $A 1$ is the backward facing side of $R 1$ or a new ring at larger separation from the star. ALMA observations might allow us to directly image the thickness of the gas disk, as was done for HD 163296 by de de Gregorio-Monsalvo et al. (2013). If we detect the dust disk truncated between $r=300-360 \mathrm{au}$, and the gas disk with $H$ comparable to the thickness we derived, this would be a strong confirmation of our understanding of the disk geometry.

Nine companion candidates are detected between 2.1-8.0" with SPHERE/IRDIS. In VLT/NACO data we detect four of these nine point sources, and determine that they are not comoving, and therefore not bound to the system. Follow-up observations of the remaining five viable companion candidates need to be made to determine if they are co-moving. If cc1 indeed turns out to be orbiting the disk at 540 au and if $A 1$ is indeed showing the backward facing side of a truncated disk, RX J1615 provides the most unambiguous example of ongoing planet-disk interaction yet detected.

Acknowledgements. We thank the anonymous referee for his/her very rapid and constructive comments. Many thanks go out to the instrument scientists and operators of the ESO Paranal observatory for their support during the observations. We also thank Michiel Min for the insightful discussion about the geometry of the disk. P. Pinilla is supported by a Royal Netherlands Academy of Arts and Sciences (KNAW) professor prize. A.J. is supported by the DISCSIM project, grant agreement 341137 funded by the European Research Council under ERC-2013-ADG. M.M. and C.G. acknowledge the German science foundation for support in the programme MU2695/131. This research has made use of the SIMBAD database, operated at the CDS, Strasbourg, France. NASA's Astrophysics Data System Bibliographic Services has been very useful for this research. SPHERE is an instrument designed and built by a consortium consisting of IPAG (Grenoble, France), MPIA (Heidelberg, Germany), LAM (Marseille, France), LESIA (Paris, France), Laboratoire Lagrange (Nice, France), INAF - Osservatorio di Padova (Italy), Observatoire de Genève (Switzerland), ETH Zurich (Switzerland), NOVA (The Netherlands), ONERA (France), and ASTRON (The Netherlands) in collaboration with ESO. SPHERE was funded by ESO, with additional contributions from the CNRS (France), MPIA (Germany), INAF (Italy), FINES (Switzerland) and NOVA (The Netherlands). SPHERE also received funding from the European Commission Sixth and Seventh Framework Programs as part of the Optical Infrared Coordination Network for Astronomy (OPTICON) under grant number RII3-Ct-2004-001566 for FP6 (2004-2008), grant number 226604 for FP7 (2009-2012), and grant number 312430 for FP7 (2013-2016).

\section{References}

Alexander, R. D., \& Armitage, P. J. 2009, ApJ, 704, 989

Allard, F., Homeier, D., \& Freytag, B. 2012, Philos. Trans. Roy. Soc. London Ser. A, 370, 2765

ALMA Partnership, Brogan, C. L., Pérez, L. M., et al. 2015, ApJ, 808, L3 Andrews, S. M., Wilner, D. J., Espaillat, C., et al. 2011, ApJ, 732, 42 Andrews, S. M., Wilner, D. J., Zhu, Z., et al. 2016, ApJ, 820, L40 Avenhaus, H., Quanz, S. P., Schmid, H. M., et al. 2014, ApJ, 781, 87 Baraffe, I., Chabrier, G., Barman, T. S., Allard, F., \& Hauschildt, P. H. 2003 A\&A, 402, 701

Benisty, M., Juhasz, A., Boccaletti, A., et al. 2015, A\&A, 578, L6

Beuzit, J.-L., Feldt, M., Dohlen, K., et al. 2008, in Ground-based and Airborne Instrumentation for Astronomy II, Proc. SPIE, 7014, 701418

Bianchi, L., Herald, J., Efremova, B., et al. 2011, Ap\&SS, 335, 161

Biller, B. A., Liu, M. C., Rice, K., et al. 2015, MNRAS, 450, 4446

Boccaletti, A., Abe, L., Baudrand, J., et al. 2008, in Adaptive Optics Systems, Proc. SPIE, 7015, 70151

Bouvier, J., Lanzafame, A. C., Venuti, L., et al. 2016, A\&A, 590, A78

Butters, O. W., West, R. G., Anderson, D. R., et al. 2010, A\&A, 520, L10 Canovas, H., Rodenhuis, M., Jeffers, S. V., Min, M., \& Keller, C. U. 2011, A\&A, 531, A102

Claudi, R. U., Turatto, M., Gratton, R. G., et al. 2008, in Ground-based and Airborne Instrumentation for Astronomy II, Proc. SPIE, 7014, 70143 
Cutri, R. M., Skrutskie, M. F., van Dyk, S., et al. 2003, 2MASS All Sky Catalog of point sources

Cutri, R. M., et al. 2013, VizieR Online Data Catalog, II/328

de Boer, J., Girard, J. H., Canovas, H., et al. 2016, MNRAS, submitted [arXiv: 1610.06609 ]

de Gregorio-Monsalvo, I., Ménard, F., Dent, W., et al. 2013, A\&A, 557, A133

de Juan Ovelar, M., Pinilla, P., Min, M., Dominik, C., \& Birnstiel, T. 2016, MNRAS, 459, L85

Devillard, N. 2001, in Astronomical Data Analysis Software and Systems X, eds

F. R. Harnden, Jr., F. A. Primini, \& H. E. Payne, ASP Conf. Ser., 238, 525

Dodson-Robinson, S. E., \& Salyk, C. 2011, ApJ, 738, 131

Dohlen, K., Langlois, M., Saisse, M., et al. 2008, in Ground-based and Airborne Instrumentation for Astronomy II, Proc. SPIE, 7014, 70143L

Dong, R., Fung, J., \& Chiang, E. 2016, ApJ, 826, 75

Fusco, T., Sauvage, J. F., Petit, C., et al. 2014, in SPIE Astronomical Telescopes + Instrumentation, eds. E. Marchetti, L. M. Close, \& J.-P. Véran, SPIE, 9148, 91481

Galli, P. A. B., Bertout, C., Teixeira, R., \& Ducourant, C. 2013, A\&A, 558, A77

Ginski, C., Schmidt, T. O. B., Mugrauer, M., et al. 2014, MNRAS, 444, 2280

Ginski, C., Stolker, T., Pinilla, P., et al. 2016, A\&A, 595, A112

Girard, J. H. V., Kasper, M., Quanz, S. P., et al. 2010, in SPIE Conf. Ser., 7736, $77362 \mathrm{~N}$

Grady, C. A., Polomski, E. F., Henning, T., et al. 2001, AJ, 122, 3396

Grady, C. A., Muto, T., Hashimoto, J., et al. 2013, ApJ, 762, 48

Helou, G., \& Walker, D. W. 1988, Infrared astronomical satellite (IRAS) catalogs and atlases, The small scale structure catalog, 1

Hollenbach, D., \& Gorti, U. 2005, in Protostars and Planets V Posters, 1286, 8433

Horne, J. H., \& Baliunas, S. L. 1986, ApJ, 302, 757

Hugot, E., Ferrari, M., El Hadi, K., et al. 2012, A\&A, 538, A139

Ishihara, D., Onaka, T., Kataza, H., et al. 2010, A\&A, 514, A1

Kraus, A. L., Ireland, M. J., Martinache, F., \& Lloyd, J. P. 2008, ApJ, 679, 762

Krautter, J., Wichmann, R., Schmitt, J. H. M. M., et al. 1997, A\&AS, 123, 329

Kuhn, J. R., Potter, D., \& Parise, B. 2001, ApJ, 553, L189

Lagage, P.-O., Doucet, C., Pantin, E., et al. 2006, Science, 314, 621

Langlois, M., Dohlen, K., Vigan, A., et al. 2014, in Ground-based and Airborne Instrumentation for Astronomy V, Proc. SPIE, 9147, 91471

Lenzen, R., Hartung, M., Brandner, W., et al. 2003, in Astronomical Telescopes and Instrumentation, eds. M. Iye, \& A. F. M. Moorwood, SPIE, 4841, 944

Maire, A.-L., Bonnefoy, M., Ginski, C., et al. 2016, A\&A, 587, A56

Makarov, V. V. 2007, ApJ, 658, 480

Manara, C. F., Testi, L., Natta, A., et al. 2014, A\&A, 568, A18

Marois, C., Lafrenière, D., Doyon, R., Macintosh, B., \& Nadeau, D. 2006, ApJ, 641, 556

Marois, C., Correia, C., Galicher, R., et al. 2014, in Adaptive Optics Systems IV, Proc. SPIE, 9148, 91480

Martinez, P., Dorrer, C., Aller-Carpentier, E., et al. 2009, The Messenger, 137, 18

Mayama, S., Hashimoto, J., Muto, T., et al. 2012, ApJ, 760, L26

Mazoyer, J., Boccaletti, A., Choquet, É., et al. 2016, ApJ, 818, 150

Merín, B., Brown, J. M., Oliveira, I., et al. 2010, ApJ, 718, 1200

Messina, S., Desidera, S., Turatto, M., Lanzafame, A. C., \& Guinan, E. F. 2010 A\&A, 520, A15

Milli, J., Mouillet, D., Lagrange, A.-M., et al. 2012, A\&A, 545, A111

Min, M., Canovas, H., Mulders, G. D., \& Keller, C. U. 2012, A\&A, 537, A75

Ochsenbein, F., Bauer, P., \& Marcout, J. 2000, A\&AS, 143, 23

Pavlov, A., Möller-Nilsson, O., Feldt, M., et al. 2008, in Advanced Software and Control for Astronomy II, Proc. SPIE, 7019, 701939

Pecaut, M. J., \& Mamajek, E. E. 2013, ApJS, 208, 9

Perrot, C., Boccaletti, A., Pantin, E., et al. 2016, A\&A, 590, L7

Pinilla, P., Benisty, M., \& Birnstiel, T. 2012, A\&A, 545, A81

Pinilla, P., de Boer, J., Benisty, M., et al. 2015, A\&A, 584, L4

Pinilla, P., Flock, M., de Juan Ovelar, M., \& Birnstiel, T. 2016, A\&A, in press, DOI: $10.1051 / 0004-6361 / 201628441$

Pojmanski, G. 1997, Acta Astron., 47, 467

Rapson, V. A., Kastner, J. H., Millar-Blanchaer, M. A., \& Dong, R. 2015, ApJ, 815, L26

Roberts, D. H., Lehar, J., \& Dreher, J. W. 1987, AJ, 93, 968

Rousset, G., Lacombe, F., Puget, P., et al. 2003, in Astronomical Telescopes and Instrumentation, eds. P. L. Wizinowich, \& D. Bonaccini, SPIE , 140
Scargle, J. D. 1982, ApJ, 263, 835

Schmid, H. M., Joos, F., \& Tschan, D. 2006, A\&A, 452, 657

Schmid, H. M., Downing, M., Roelfsema, R., et al. 2012, in Ground-based and Airborne Instrumentation for Astronomy IV, Proc. SPIE, ETH Zürich, Switzerland

Siess, L., Dufour, E., \& Forestini, M. 2000, A\&A, 358, 593

Soummer, R., Pueyo, L., \& Larkin, J. 2012, ApJ, 755, L28

Strom, K. M., Strom, S. E., Edwards, S., Cabrit, S., \& Skrutskie, M. F. 1989, AJ, 97, 1451

Thalmann, C., Schmid, H. M., Boccaletti, A., et al. 2008, in Ground-based and Airborne Instrumentation for Astronomy II, Proc. SPIE, 7014, 70143

Thi, W.-F., Pinte, C., Pantin, E., et al. 2014, A\&A, 561, A50

Tuthill, P. G., Monnier, J. D., Danchi, W. C., Wishnow, E. H., \& Haniff, C. A. 2000, PASP, 112, 555

van Boekel, R., Henning, T., Menu, J., et al. 2016, ApJ, submitted [arXiv: 1610.08939]

van der Marel, N., van Dishoeck, E. F., Bruderer, S., et al. 2013, Science, 340, 1199

van der Marel, N., van Dishoeck, E. F., Bruderer, S., Pérez, L., \& Isella, A. 2015, A\&A, 579, A106

Vigan, A., Langlois, M., Moutou, C., \& Dohlen, K. 2008, A\&A, 489, 1345

Vigan, A., Moutou, C., Langlois, M., et al. 2010, MNRAS, 407, 71

Vigan, A., Patience, J., Marois, C., et al. 2012, A\&A, 544, A9

Vigan, A., Bonnefoy, M., Ginski, C., et al. 2016, A\&A, 587, A55

Wahhaj, Z., Cieza, L., Koerner, D. W., et al. 2010, ApJ, 724, 835

Weinberger, A. J., Becklin, E. E., Schneider, G., et al. 1999, ApJ, 525, L53

Wichmann, R., Covino, E., Alcalá, J. M., et al. 1999, MNRAS, 307, 909

Zurlo, A., Vigan, A., Mesa, D., et al. 2014, A\&A, 572, A85

1 Leiden Observatory, Leiden University, PO Box 9513, 2300 RA Leiden, The Netherlands e-mail: deboer@strw. leidenuniv.nl

2 Aix-Marseille Université, CNRS, LAM (Laboratoire d'Astrophysique de Marseille) UMR 7326, 13388 Marseille, France

3 Université Grenoble Alpes, IPAG, 38000 Grenoble, France

4 LESIA, CNRS, Observatoire de Paris, Université Paris Diderot, UPMC, 5 place J. Janssen, 92190 Meudon, France

5 Institute of Astronomy, Madingley Road, Cambridge CB3 OHA, UK

6 Max-Planck-Institut fuer Astronomie, Koenigstuhl 17, 69117 Heidelberg, Germany

7 INAF-Osservatorio Astronomico di Padova, Vicolo dell'Osservatorio 5, 35122 Padova, Italy

8 INAF Catania Astrophysical Observatory, via S. Sofia 78, 95123 Catania, Italy

9 Observatoire de Genève, Université de Genève, 51 chemin des Maillettes, 1290 Versoix, Switzerland

10 European Southern Observatory, Alonso de Cordova 3107, Casilla 19001 Vitacura, Santiago 19, Chili

11 Observatoire de Lyon, Centre de Recherche Astrophysique de Lyon, École Normale Supérieure de Lyon, CNRS, Université Lyon 1, UMR 5574, 9 avenue Charles André, 69230 Saint-Genis Laval, France

12 Sterrenkundig Instituut Anton Pannekoek, Science Park 904, 1098 XH Amsterdam, The Netherlands

13 Department of Physics \& Astronomy, Rice University, 6100 Main Street, Houston, TX 77005, USA

14 Department of Astronomy, Stockholm University, AlbaNova University Center, 10691 Stockholm, Sweden

15 Astrophysical Institute and University Observatory Jena, Schillergäßchen 2, 07745 Jena, Germany

16 Instituto de Física y Astronomía, Facultad de Ciencias, Universidad de Valparaíso, Av. Gran Bretaña 1111, Playa Ancha, Valparaíso, Chile

17 Núcleo de Astronomía, Facultad de Ingeniería, Universidad Diego Portales, Av. Ejercito 441, Santiago, Chile 


\section{Appendix A: Stellar properties}

\section{Rotation period}

We have taken advantage of publicly available SuperWASP (Butters et al. 2010) photometry time series data, collected during 2004-2006, to determine the rotation period. This consists of $12684 \mathrm{~V}$-band measurements with an average photometric precision $\sigma=0.018 \mathrm{mag}$. After the removal of outliers and low-precision measurements from the time series by applying a moving boxcar filter with $3 \sigma$ threshold, we averaged consecutive data collected within $1 \mathrm{~h}$. Finally we were left with 545 averaged magnitudes with an associated standard deviation $\sigma=0.010$ mag for the subsequent analysis. To search for the rotation period of RX J1615, we applied the Lomb-Scargle method (LS, Scargle 1982), with the prescription of Horne \& Baliunas (1986), as well as the Clean (Roberts et al. 1987) periodogram analyses of the data. In all three SuperWASP observing seasons (2004, 2005 and 2006) we have searched in the period range $0.1-100 \mathrm{~d}$, and find with both LS and Clean the same rotation period $P=5.719 \pm 0.014 \mathrm{~d}$, which is the first determination for the rotation period of $\mathrm{RXJ} 1615$. Although other peaks due to observation timings and beat frequencies are present in the periodogram, we do not detect any other significant peaks of interest.

RXJ1615 was also observed by the All Sky Automated Survey (ASAS, Pojmanski 1997) in the years 2001-2009. Despite the lower photometric precision, our LS and Clean analyses allowed us to measure the same $P=5.72 \pm 0.01 \mathrm{~d}$ rotation period and a peak-to-peak light curve amplitude of $\Delta V=0.12 \mathrm{mag}$.

\section{Spectral type and temperature}

In the literature, the spectral type is found in the range from K4 (Merín et al. 2010, based on Spitzer/IRS spectra) to K7 (Manara et al. 2014, using spectroscopy with VLT/X-shooter), with Wichmann et al. (1999, with visible and NIR photometry) and Krautter et al. (1997, based on visible light spectroscopy) favoring a K5 classification. Using the sequence of intrinsic colors and temperatures of pre-main sequence stars by Pecaut \& Mamajek (2013) and comparing it to the measured colors by Makarov (2007) we find that the photometric colors are fully compatible with a K5-K6 star suffering a small or even negligible amount of reddening. The colors of a $\mathrm{K} 4$ star would instead indicate a reddening of about $E(B-V)=0.15$ mag while a K7 star is expected to have redder colors than those observed. Previous estimates of extinction span between $A_{V}=0 \mathrm{mag}$ (Manara et al. 2014) to $A_{V}=1.1 \mathrm{mag}$ (Wahhaj et al. 2010) with an intermediate value $A_{V}=0.4 \mathrm{mag}$ by Andrews et al. (2011).

Assuming the spectral type K5-K6 and a low amount of reddening $(E(B-V)<0.15 \mathrm{mag})$, the corresponding effective temperatures in the scale by Pecaut \& Mamajek (2013) are $T_{\text {eff }}=4140-4020 \mathrm{~K}$ for $\mathrm{K} 5$ and $\mathrm{K} 6$ spectral types respectively. We have also used the available optical, near-IR, and IR photometry to build the observed SED (Fig. A.1). We have obtained the far ultra-violet magnitudes from the GALEX catalogs (Bianchi et al. 2011); the UBVRI magnitudes are taken from Makarov (2007); Denis $I J K$ magnitudes from DENIS Catalogue (Ochsenbein et al. 2000); JHK magnitudes from the 2MASS project (Cutri et al. 2003); W1 - W4 magnitudes from the WISE project (Cutri et al. 2013); IRAS magnitudes from the IRAS Catalog of Point Sources (Helou \& Walker 1988); AKARI magnitudes from AKARI/IRC mid-IR all-sky Survey (Ishihara et al. 2010). The SED was fit with a grid of theoretical spectra from the BT-NextGen Model (Allard et al. 2012) and the best fit, shown in Fig. A.1 is obtained with a model of $T_{\text {eff }}=4100 \pm 50 \mathrm{~K}$, when adopting $d=185 \mathrm{pc}$ and $E(B-V)=0.00 \mathrm{mag}$. We note the presence of a significant $\mathrm{UV}$ and IR excess which is common when there is an associated disk. Combining our two estimates we conservatively take the temperature to be $T_{\text {eff }}=4100 \pm 100 \mathrm{~K}$.

\section{Stellar rotation axis inclination}

Using the brightest visual magnitude $V=12.0 \mathrm{mag}$ from the ASAS time series, $d=185 \mathrm{pc}, A_{V}=0.00 \mathrm{mag}$, and bolometric correction from Pecaut $\&$ Mamajek (2013) tables, we derive the stellar luminosity $L_{\star}=1.07 \pm 0.11 L_{\odot}$. For $T_{\text {eff }}=4100 \mathrm{~K}$, we derive the stellar radius $R_{\star}=2.05 \pm 0.21 R_{\odot}$. With the stellar radius and the rotation period determined above we can compute the rotational velocity at the stellar surface $v=18.1 \mathrm{~km} \mathrm{~s}^{-1}$. From the projected rotational velocity $v \sin i=13.0 \mathrm{~km} \mathrm{~s}^{-1}$ (Wichmann et al. 1999), we derive the inclination of the rotation axis $i=46 \pm 6^{\circ}$, which is compatible within errors with the inclination $i=45 \pm 5^{\circ}$ of the disk derived by van der Marel et al. (2015, and priv. comm.). Therefore, we infer that the disk is coplanar with the stellar equatorial plane. If we instead assume an extinction $A_{V}=0.4 \mathrm{mag}$ (Andrews et al. 2011) to determine $L_{\star}$ and $R_{\star}$, the inferred inclination $i=37 \pm 6^{\circ}$.

\section{Age, mass, and distance}

A comparison of the rotation period of RXJ1615 with the distribution of rotation periods of stellar associations of known age helps us to constrain the age. We find that the rotation period $P=5.719 \mathrm{~d}$ is longer than for members of the TW Hya association $(P=4 \mathrm{~d}$, age $=9 \mathrm{Myr}$, Messina et al. 2010). Considering that RXJ1615 is a pre-main sequence star whose radius is still contracting and its rotation still spinning up, we can state that RX J1615 is not older than $~ 9$ Myr. The measured stellar lithium (Li) equivalent width of $558 \mathrm{~m} \AA$ (Wichmann et al. 1999) fits very well into the Li distribution of the 5 Myr NGC 2264 open cluster (Bouvier et al. 2016) leading us to expect an even younger age compatible with that expected for a CTTS with a gas-rich disk.

Makarov (2007) classified the star as a member of the Lupus association, deriving a kinematic distance of $184 \mathrm{pc}$. They also noted a group of stars that appear younger ( $\sim 1$ Myr as opposed to $\sim 5 \mathrm{Myr}$ ) and at a greater distance than the bulk of the members of the association. However, the star is not considered in the most recent study of Lupus (Galli et al. 2013). 
A\&A 595, A114 (2016)

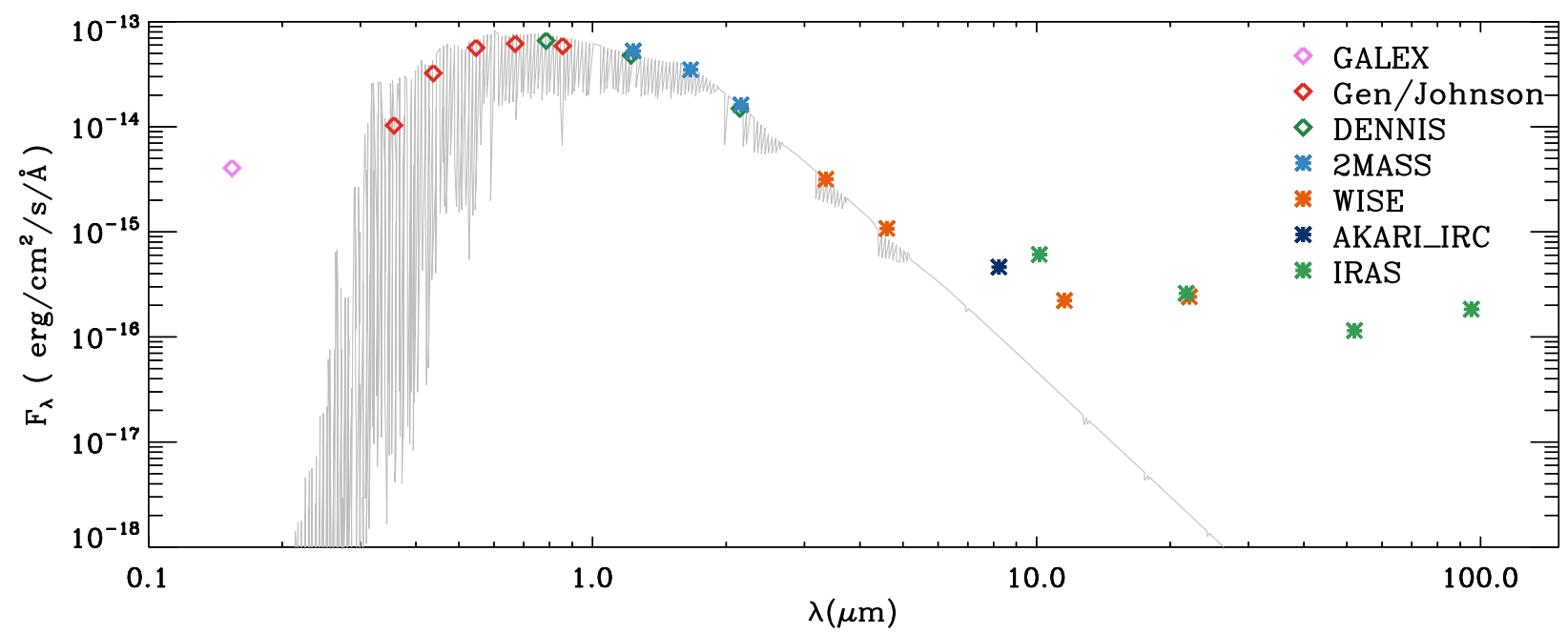

Fig. A.1. Spectral energy distributions (SED, see legend) and our best fit BT-NextGen model (grey solid line, $T_{\text {eff }}=4100 \mathrm{~K}$ ) for RX J1615. 